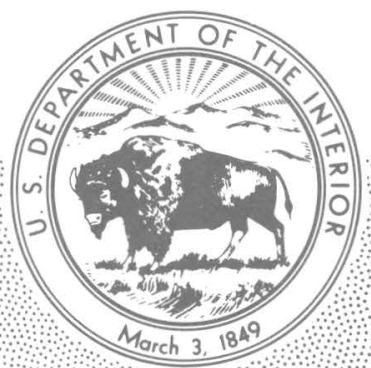

\title{
Catalogue of U.S. Geological Survey Strong-Motion Records, 1988
}




\section{AVAILABILITY OF BOOKS AND MAPS OF THE U.S. GEOLOGICAL SURVEY}

Instructions on ordering publications of the U.S. Geological Survey, along with prices of the last offerings, are given in the current-year issues of the monthly catalog "New Publications of the U.S. Geological Survey." Prices of available U.S. Geological Survey publications released prior to the current year are listed in the most recent annual "Price and Availability List." Publications that are listed in various U.S. Geological Survey catalogs (see back inside cover) but not listed in the most recent annual "Price and Availability List" are no longer available.

Prices of reports released to the open files are given in the listing "U.S. Geological Survey Open-File Reports," updated monthly, which is for sale in microfiche from the U.S. Geological Survey, Books and Open-File Reports Section, Federal Center, Box 25425, Denver, CO 80225. Reports released through the NTIS may be obtained by writing to the National Technical Information Service, U.S. Department of Commerce, Springfield, VA 22161; please include NTIS report number with inquiry.

Order U.S. Geological Survey publications by mail or over the counter from the offices given below.

\section{BY MAIL}

\section{Books}

Professional Papers, Bulletins, Water-Supply Papers, Techniques of Water-Resources Investigations, Circulars, publications of general interest (such as leaflets, pamphlets, booklets), single copies of Earthquakes \& Volcanoes, Preliminary Determination of Epicenters, and some miscellaneous reports, including some of the foregoing series that have gone out of print at the Superintendent of Documents, are obtainable by mail from

\section{U.S. Geological Survey, Books and Open-File Reports Federal Center, Box 25425 Denver, CO 80225}

Subscriptions to periodicals (Earthquakes \& Volcanoes and Preliminary Determination of Epicenters) can be obtained ONLY from the

\section{Superintendent of Documents \\ Government Printing Office \\ Washington, D.C. 20402}

(Check or money order must be payable to Superintendent of Documents.)

\section{Maps}

For maps, address mail orders to

\section{U.S. Geological Survey, Map Distribution \\ Federal Center, Box 25286 \\ Denver, CO 80225}

Residents of Alaska may order maps from

\author{
Alaska Distribution Section, U.S. Geological Survey, \\ New Federal Building - Box 12 \\ 101 Twelfth Ave., Fairbanks, AK 99701
}

\section{OVER THE COUNTER}

\section{Books}

Books of the U.S. Geological Survey are available over the counter at the following Geological Survey Public Inquiries Offices, all of which are authorized agents of the Superintendent of Documents:

- WASHINGTON, D.C.--Main Interior Bldg., 2600 corridor, 18th and C Sts., NW.

- DENVER, Colorado--Federal Bldg., Rm. 1691961 Stout St.

- LOS ANGELES, California--Federal Bldg., Rm. 7638, 300 N. Los Angeles St.

- MENLO PARK, California--Bldg. 3 (Stop 533), Rm. 3128, 345 Middlefield Rd.

- RESTON, Virginia--503 National Center, Rm. 1C402, 12201 Sunrise Valley Dr.

- SALT LAKE CITY, Utah--Federal Bldg., Rm. 8105, 125 South State St.

- SAN FRANCISCO, California--Customhouse, Rm. 504, 555 Battery St.

- SPOKANE, Washington--U.S. Courthouse, Rm. 678, West 920 Riverside Ave..

- ANCHORAGE, Alaska--Rm. 101, 4230 University Dr.

- ANCHORAGE, Alaska--Federal Bldg, Rm. E-146, 701 C St.

Maps

Maps may be purchased over the counter at the U.S. Geological Survey offices where books are sold (all addresses in above list) and at the following Geological Survey offices:

- ROLLA, Missouri--1400 Independence Rd.

- DENVER, Colorado--Map Distribution, Bldg. 810, Federal Center

- FAIRBANKS, Alaska--New Federal Bldg., 101 Twelfth Ave. 


\section{Catalogue of U.S. Geological Survey Strong-Motion Records, 1988}

Compiled by JOSEPHINE C. SWITZER and RONALD L. PORCELLA 
U.S. DEPARTMENT OF THE INTERIOR

MANUEL LUJAN, JR., Secretary

\section{U.S. GEOLOGICAL SURVEY}

Dallas L. Peck, Director

Any use of trade, product, or firm names

in this publication is for descriptive purposes only

and does not imply endorsement by the U.S. Government

UNITED STATES GOVERNMENT PRINTING OFFICE, WASHINGTON : 1990

For sale by the

Books and Open-File Reports Section

U.S. Geological Survey

Federal Center, Box 25425

Denver, CO 80225

Library of Congress Cataloging Card No. 83-600616 


\section{PREFACE}

The first seismic engineering program in the United States was administered by the Seismological Field Survey (SFS) of the Coast and Geodetic Survey. This program was begun in 1931 and essentially remained the responsibility of the SFS until 1973, when the U.S. Geological Survey (USGS) assimilated the program into its National Earthquake Hazards Reduction Program. The current Federal seismic engineering program operates the National Cooperative Strong-Motion Network (NCSMN) with nearly 1,000 stations in 40 States and Puerto Rico. This network is administered by the USGS in cooperation with both private industry and numerous Federal, State, and local agencies and organizations. Major contributors include the Army Corps of Engineers, the Veterans Administration, and the Metropolitan Water District of Southern California. Primary objectives of the program are to record strong ground motions and the response of representative engineered structures during moderate to large earthquakes, and to disseminate the resultant data and information about the records, sites, and structures to the earthquake engineering research and design community.

This catalogue continues in a revised format the yearly publication "Strong-Motion Program Report, January-December [year]"; it is a continuation of the table 1 summary of accelerograms recovered at NCSMN stations that had been published in that format since 1974. This report includes all accelerograms recovered during 1988. Unless otherwise noted, event data are from the "Preliminary Determination of Epicenters," published monthly by the USGS. 



\section{CONTENTS}

Preface III

Introduction 1

References 1

TABLE

1. Catalogue of National Cooperative Strong-Motion Network accelerograph records recovered during 19883 



\title{
Catalogue of U.S. Geological Survey Strong-Motion Records, 1988
}

\author{
Compiled by Josephine C. Switzer and Ronald L. Porcella
}

\section{INTRODUCTION}

The National Cooperative Strong-Motion Network (NCSMN), with stations in 40 states and Puerto Rico, produced nearly 300 accelerograph records at recording sites in California, Alaska, and Hawaii during the period January-December 1988; more than 80 percent were recorded in California. The network has produced an annual average of 250 records for the years 1974 through 1987. Although there were no significant strongmotion events recorded during 1988 , there were nine notable earthquakes in the magnitude range 4.6 to 5.6 that produced several interesting suites of records at a total of 87 NCSMN stations. Eight of these events occurred in California and one in southern Alaska.

An $M_{\mathrm{L}}=4.7$ earthquake in the Imperial Valley of California triggered 11 stations on January 28 (G.m.t.); the event was located on the Superstition Hills fault with an epicenter very near the Parachute Test Site recording station, where the maximum horizontal ground acceleration reached $0.17 \mathrm{~g}$.

On February 11 an $M_{\mathrm{L}}=4.7$ aftershock of the October 1,1987 , Whittier Narrows earthquake triggered 19 accelerograph stations in the Los Angeles region. This aftershock was located approximately $3 \mathrm{~km}$ northeast of the main shock epicenter and produced strong-motion recordings at eight buildings, nine dam/reservoir facilities, one hospital, and one ground site (Acosta and others, 1988). Peak horizontal motion exceeded $0.2 \mathrm{~g}$ at three locations: the basement of a 10-story building in Whittier, the upstream station at Whittier Narrows Dam, and the abutment station at Garvey Reservoir.

On February 20, an $M_{\mathrm{L}}=5.3$ earthquake in central California triggered three accelerographs in the Hollister region (Salsman and Forshee, 1988). Peak recorded ground motion reached $0.21 \mathrm{~g}$ on one of 13 horizontal components operating at the Hollister Differential Array.

Two earthquakes along the Calaveras fault zone in northern California on June 13 and November 10 each triggered seven strong-motion stations at epicentral distances in the range 8 to $28 \mathrm{~km}$. The $M_{\mathrm{L}}=5.4$ June 13 event occurred at a depth of $7 \mathrm{~km}$ and produced a peak horizontal ground acceleration of $0.11 \mathrm{~g}$ in Fremont. The $M_{\mathrm{L}}=4.8$ November 10 event occurred at a 9-km depth and produced a peak horizontal ground acceleration of $0.17 \mathrm{~g}$ at the Interstate 280/101 Interchange abutment site in San Jose. The epicentral distances for these two peak recordings were 22 and $9 \mathrm{~km}$, respectively (Salsman and Switzer, 1990).

On June 26, a magnitude 4.6 $\left(M_{\mathrm{L}}\right)$ earthquake near Upland in southern California produced significant ground motions at three of four stations triggered by this event. Peak motions and their locations were $0.12 \mathrm{~g}$ at Weymouth Filter Plant, $0.23 \mathrm{~g}$ at Live Oak Reservoir Abutment, and $0.31 \mathrm{~g}$ at San Antonio Dam Downstream.

An $M_{\mathrm{L}}=4.9$ earthquake on December 3 near Pasadena triggered strong-motion instrumentation at 23 stations in the Los Angeles region; epicentral distances were in the range 8 to $42 \mathrm{~km}$ (Acosta and Johnson, 1989). Acceleration records were recovered from 45 instruments located at five ground sites, four dams, one reservoir, two filter plants, three hospitals, and eight buildings. Maximum horizontal ground acceleration was $0.12 \mathrm{~g}$, recorded at two stations in the vicinity of Los Angeles.

Manuscript approved for publication, September 21, 1990. 
On December 16 an $M_{\mathrm{L}}=4.8$ earthquake in southern California triggered seven stations in the North Palm Springs region. Peak recorded ground acceleration was $0.15 \mathrm{~g}$ at the Whitewater Trout Farm station, located on rock approximately $5 \mathrm{~km}$ north of the San Andreas fault zone.

\section{REFERENCES}

Acosta, A.V., Nielson, J.D., and Switzer, J.C., 1988, Strong-motion data from the Whittier Narrows aftershock of February 11, 1988: U.S. Geological Survey Open-File Report 88-357, 27 p.

Acosta, A.V., and Johnson, D.A., 1989, Strong-motion data from the Pasadena, California, earthquake of December 3, 1988: U.S. Geological Survey Open-File Report 89-203, 57 p.

Salsman, M.J., and Forshee, R.D., 1988, Strong-motion data from the Hollister earthquake of February 20, 1988: U.S. Geological Survey Open-File Report 88-565, 12 p.

Salsman, M.J., and Switzer, J.C., in press, Strong-motion records from the Calaveras fault earthquakes of June 13, 1988, November 10, 1988, and April 3, 1989: U.S. Geological Survey Open-File Report 90-481, 36 p. 


\section{Catalogue of Mational Cooperative Strong-Motion Network accelerograph records recovered during 1988}

[Station owners: ACOE, U.S. Army Corps of Engineers; BECH, Bechtel Power Corporation; CLA, City of Los Angeles; GLDN, U.S. Geological Survey, Golden, Colorado; JCG, JCG Finance Corporation of America; MANC, Municipality of Anchorage, Alaska; MWD, Metropolitan Water District of Southern Calif.; OWNR, Owner of building; UCB, University of California at Berkeley; USGS, U.S. Geological Survey; VA, U.S. Veterans Administration. Instrument trigger time in minutes and seconds after the hour 1 isted in earthquake column. Trigger time in brackets is a $P$-wave arrival time as event occurred while instrument was operating. S-minus trigger denotes $\underline{S}$-wave-arrival-minus-trigger-time $(\underline{\mathrm{S}}-\underline{\mathrm{t}})$ or $\underline{\mathrm{S}}$-wave-minus- $\mathrm{P}$-wave-arrival-time ( $\underline{\mathrm{S}}-\underline{\mathrm{P}}$, in brackets) interval. Direction $\overline{i s}$ of case acceleration for upward trace deflection on accelerogram; horizontal components are listed as azimuth, and vertical components as "up" or "down." Maximum amplitude is peak acceleration recorded at ground level on one vertical and two orthogonal horizontal components unless otherwise noted. Duration is interval between first and last peaks of acceleration greater than $0.10 \mathrm{~g}$.

\begin{tabular}{|c|c|c|c|c|c|c|c|}
\hline Earthquake & $\begin{array}{l}\text { Station name } \\
\text { (owner) }\end{array}$ & $\begin{array}{c}\text { Station } \\
\text { location } \\
\left({ }^{\circ}\right)\end{array}$ & $\begin{array}{l}\text { Trigger } \\
\text { time }\end{array}$ & $\begin{array}{c}\text { S-minus } \\
\text { trigger } \\
(\mathrm{s})\end{array}$ & $\begin{array}{l}\text { Direction } \\
\quad(a z)\end{array}$ & $\begin{array}{l}\text { Max imum } \\
\text { ampl itude } \\
\text { (g) }\end{array}$ & $\begin{array}{c}\text { Duration } \\
(\mathrm{s})\end{array}$ \\
\hline
\end{tabular}

7 November 1987 1506:01.2 G.m.t. Central Calif. $36.583 \mathrm{~N}, 121.218 \mathrm{~W}$ Magnitude 4.0 ML

4 January 1988 1309 G.m.t. Southern Calif. Epicenter and magnitude unknown

12 January 1988 0444 G.m.t. Central Calif. Epicenter and magnitude unknown

October 198720 January 1988 Southern Cal if. Epicenter and magnitude unknown

28 January 1988 0254:02.3 G.m.t. Southern Cal if. $32.910 \mathrm{~N}, 115.680 \mathrm{~W}$ Magnitude 4.7 ML (USGS) Camera Site (USGS)

McGee Creek
Bear Valley Station $14 \quad 36.569 \mathrm{~N} \quad 06: 04.9 \quad 2.9$

$121.043 \mathrm{~W}$

Superstition Mounta in

$32.955 \mathrm{~N}$

$09: 42.6$

(2)

Note: One additional record ${ }^{1}$ recovered at Superstition Mountain Camera Site.
Crest

Downstream

Crowley Lake (USGS) (Triaxial)

McGee Creek

Crowley Lake (USGS) (Multi-channel)

166 m Downhole

35 m Downhole

1 m Downhole

Surface

\section{San Antonio Dam}

Upland

(ACOE)

Brawley Airport

Hangar

(USGS)
$37.550 \mathrm{~N} \quad 44: 20.6^{4}$

(2)

$118.811 \mathrm{~W}$

$37.550 \mathrm{~N} \quad 44: 20.6^{4}$ $118.811 \mathrm{~W}$
$34.157 \mathrm{~N}$

$117.676 \mathrm{~W}$

(3)

$32.991 \mathrm{~N}$

(3)

3.0

315
Up
225

.09
.03
.07

0.0

0.0

0.0 
Catalogue of Mational Cooperative Strong-Motion Metwork accelerograph records recovered during 1988--Continued

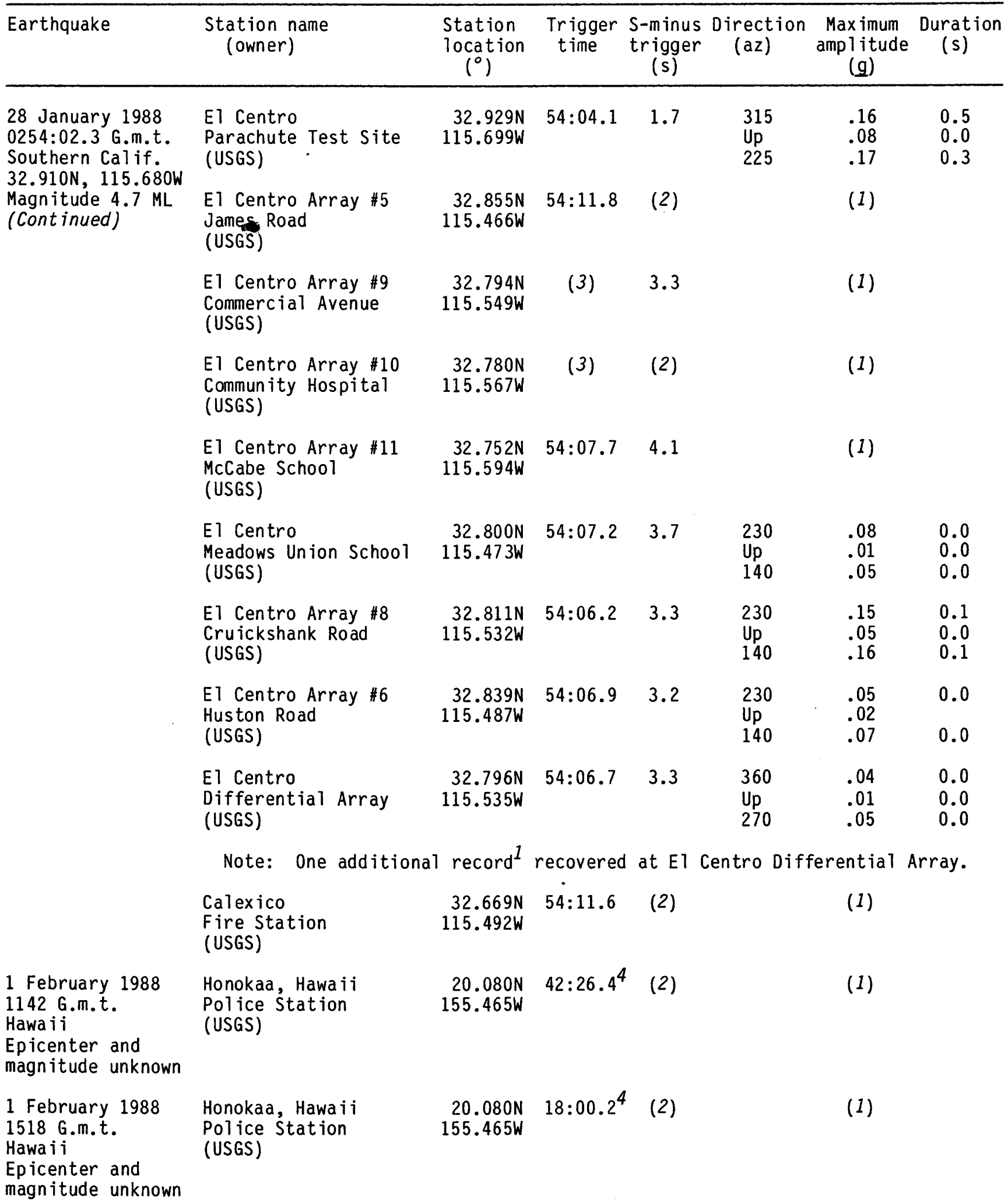


Catalogue of National Cooperative Strong-Motion Network

accelerograph records recovered during 1988--Continued

\begin{tabular}{|c|c|c|c|c|c|c|c|}
\hline Earthquake & $\begin{array}{l}\text { Station name } \\
\text { (owner) }\end{array}$ & $\begin{array}{l}\text { Station } \\
\text { location } \\
\left({ }^{\circ}\right)\end{array}$ & $\begin{array}{c}\text { Trigger } \\
\text { time }\end{array}$ & $\begin{array}{l}\text { S-minus } \\
\text { trigger } \\
\text { (s) }\end{array}$ & $\begin{array}{l}\text { Direction } \\
\quad(a z)\end{array}$ & $\begin{array}{l}\text { Maximum } D \\
\text { amplitude } \\
\text { (g) }\end{array}$ & $\begin{array}{l}\text { Duration } \\
\text { (s) }\end{array}$ \\
\hline \multirow{8}{*}{$\begin{array}{l}7 \text { February } 1988 \\
0846: 58.6 \text { G.m.t. } \\
\text { Southern Alaska } \\
60.296 \mathrm{~N}, 152.972 \mathrm{~W} \\
\text { Magnitude } 5.6 \mathrm{MB}\end{array}$} & $\begin{array}{l}\text { Anchorage } \\
\text { Fire Station \#4 } \\
\text { (MANC) }\end{array}$ & $\begin{array}{r}61.182 \mathrm{~N} \\
149.848 \mathrm{~W}\end{array}$ & (3) & 18.6 & & (1) & \\
\hline & Note: Seven additi & nal records ${ }^{1}$ & 1 recover & red at & Anchorage & Fire Station & in \#4. \\
\hline & $\begin{array}{l}\text { Anchorage } \\
\text { Fire Station \#5 } \\
\text { (MANC) }\end{array}$ & $\begin{array}{r}61.186 \mathrm{~N} \\
149.921 \mathrm{~W}\end{array}$ & (3) & (2) & & (1) & \\
\hline & $\begin{array}{l}\text { Anchorage } \\
\text { New Federal Building } \\
\text { (USGS) }\end{array}$ & $\begin{array}{r}61.216 \mathrm{~N} \\
149.883 \mathrm{~W}\end{array}$ & (3) & (2) & & (1) & \\
\hline & $\begin{array}{l}\text { Anchorage } \\
\text { Lutheran Church } \\
\text { (USGS/GLDN) }\end{array}$ & $\begin{array}{rr}61.209 \mathrm{~N} & 4 \\
149.891 \mathrm{~W} & \end{array}$ & $47: 54.4^{4}$ & (2) & & (1) & \\
\hline & $\begin{array}{l}\text { Homer } \\
\text { Airport Fire Station } \\
\text { (USGS) }\end{array}$ & $\begin{array}{r}59.64 \mathrm{~N} \\
151.50 \mathrm{~W}\end{array}$ & (3) & 14.2 & & (1) & \\
\hline & $\begin{array}{l}\text { Seward } \\
\text { Fire Station \#1 } \\
\text { (USGS) }\end{array}$ & $\begin{array}{r}60.101 \mathrm{~N} \\
149.434 \mathrm{~W}\end{array}$ & (3) & (2) & & & \\
\hline & Basement & & & & (1) & & \\
\hline \multirow{7}{*}{$\begin{array}{l}11 \text { February } 1988 \\
1525: 55.6 \mathrm{G.m.t} \text {. } \\
\text { Southern Cal if. } \\
34.080 \mathrm{~N}, 118.050 \mathrm{WL} \\
\text { Magnitude } 4.7 \mathrm{ML}\end{array}$} & $\begin{array}{l}\text { Whittier Narrows Dam } \\
\text { Pico Rivera } \\
\text { (ACOE) }\end{array}$ & $\begin{array}{r}34.020 \mathrm{~N} \\
118.053 \mathrm{~W}\end{array}$ & (3) & 2.2 & & & \\
\hline & Crest & & & & $\begin{array}{l}033 \\
\text { Up } \\
303\end{array}$ & $\begin{array}{l}.11 \\
.09 \\
.13\end{array}$ & $\begin{array}{c}1 \text { peak } \\
0.0 \\
1 \text { peak }\end{array}$ \\
\hline & Upstream & & & & $\begin{array}{l}152 \\
U p \\
062\end{array}$ & $\begin{array}{l}.24 \\
.19 \\
.24\end{array}$ & $\begin{array}{l}1.2 \\
0.7 \\
0.7\end{array}$ \\
\hline & $\begin{array}{l}\text { Garvey Reservoir } \\
\text { Monterey Park } \\
\text { (MWD) }\end{array}$ & $\begin{array}{r}34.050 \mathrm{~N} \\
118.114 \mathrm{~W}\end{array}$ & (3) & 2.5 & & & \\
\hline & Abutment Bldg. & & & & $\begin{array}{l}060 \\
\text { Up } \\
330\end{array}$ & $\begin{array}{l}.22 \\
.11 \\
.15\end{array}$ & $\begin{array}{l}0.3 \\
1 \text { peak } \\
0.6\end{array}$ \\
\hline & $\begin{array}{l}\text { Alhambra } \\
900 \mathrm{~S} \text {. Fremont } \\
\text { (USGS) }\end{array}$ & $\begin{array}{r}34.085 \mathrm{~N} \\
118.149 \mathrm{~W}\end{array}$ & (3) & 1.1 & & & \\
\hline & Basement & & & & $\begin{array}{l}090 \\
\text { Up } \\
360\end{array}$ & $\begin{array}{l}.07 \\
.03 \\
.06\end{array}$ & $\begin{array}{l}0.0 \\
0.0 \\
0.0\end{array}$ \\
\hline
\end{tabular}


Catalogue of National Cooperative Strong-Motion Network accelerograph records recovered during 1988--Continued

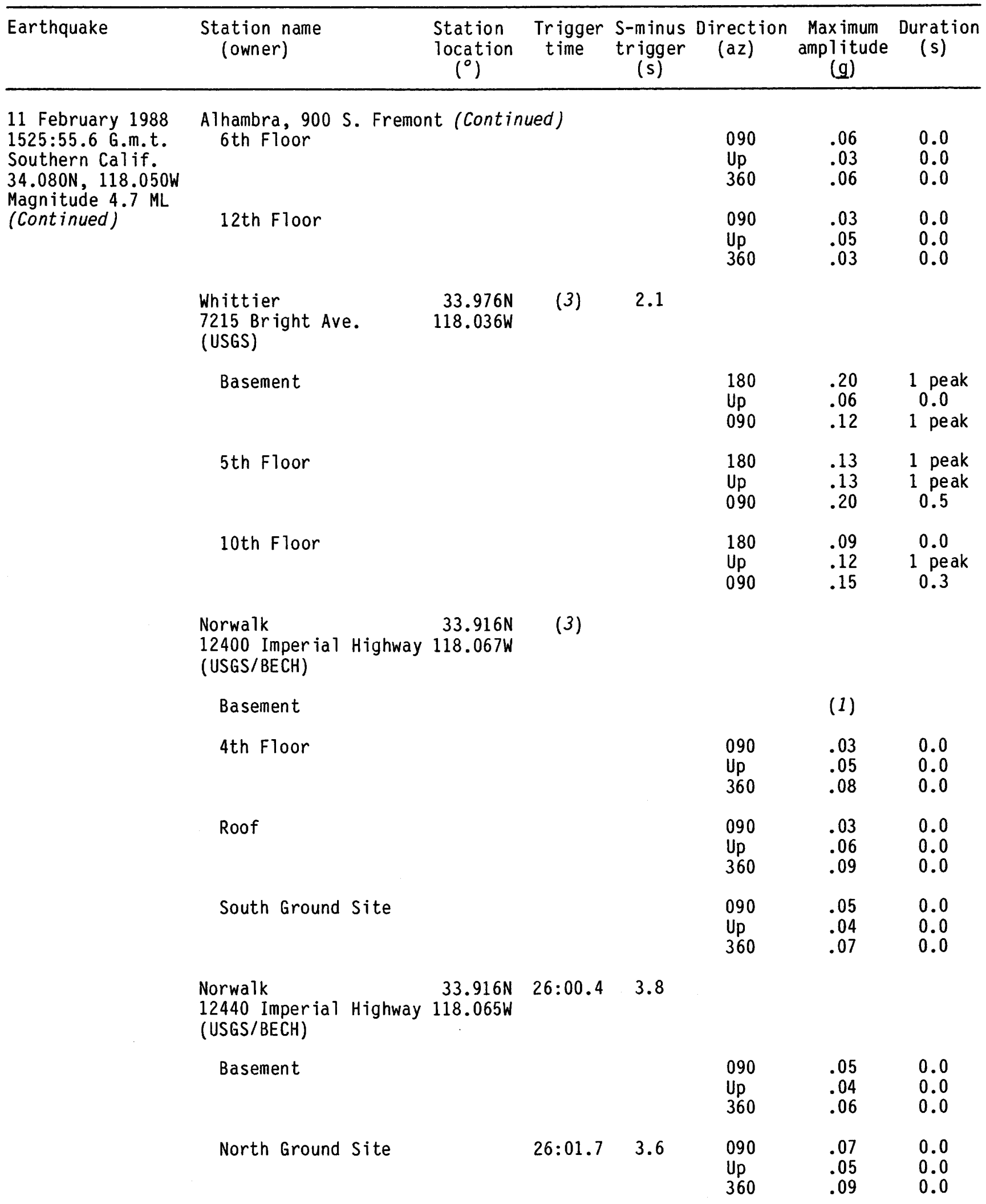


Catalogue of National Cooperative Strong-Motion Network accelerograph records recovered during 1988--Cont inued

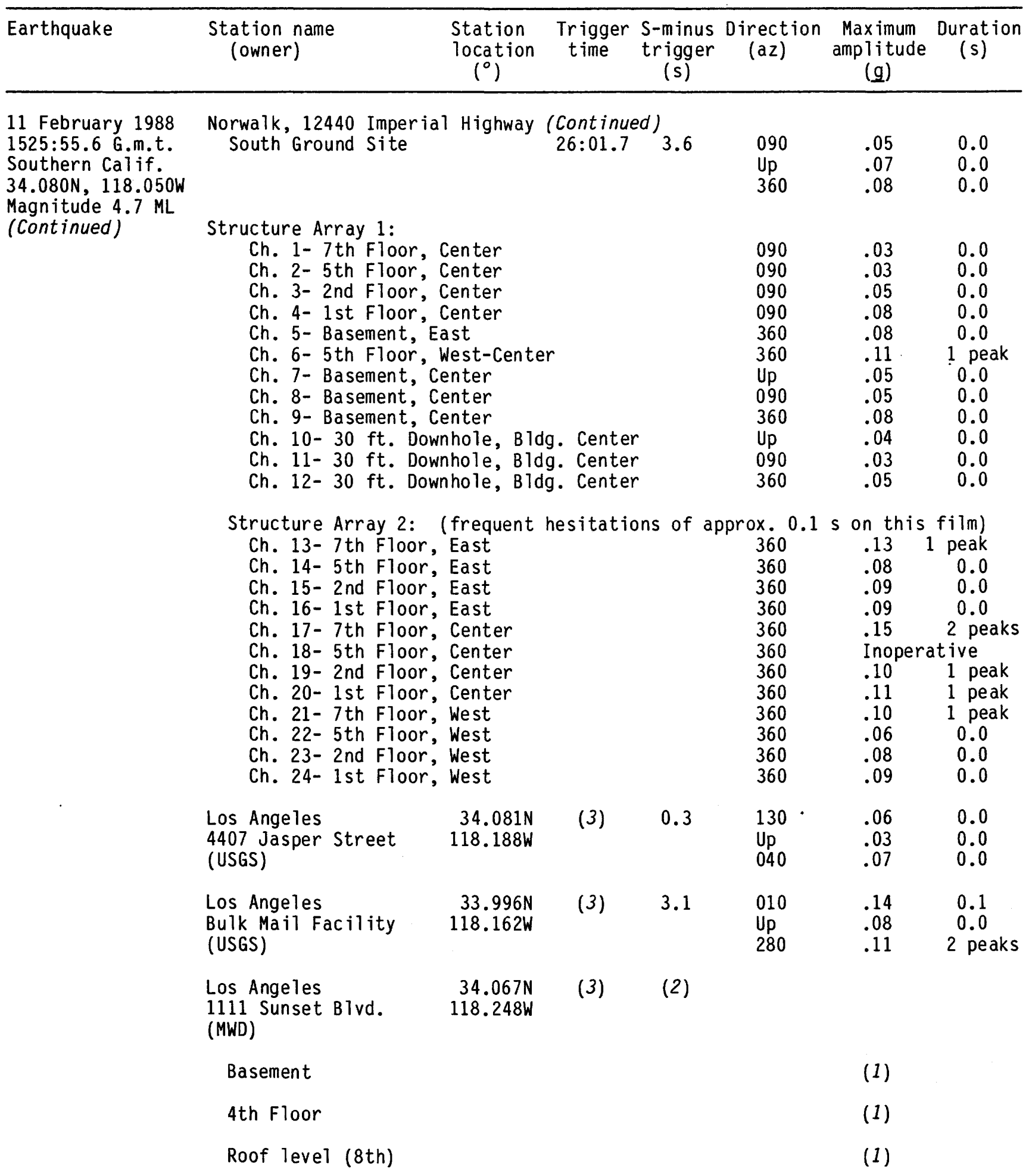


Catalogue of National Cooperative Strong-Motion Network accelerograph records recovered during 1988--Cont inued

\begin{tabular}{|c|c|c|c|c|c|c|c|}
\hline Earthquake & $\begin{array}{l}\text { Station name } \\
\text { (owner) }\end{array}$ & $\begin{array}{l}\text { Station } \\
\text { location } \\
\left({ }^{\circ}\right)\end{array}$ & $\begin{array}{l}\text { Trigger } \\
\text { time }\end{array}$ & $\begin{array}{l}\text { S-minus } \\
\text { trigger } \\
(\mathrm{s})\end{array}$ & $\begin{array}{l}\text { Direction } \\
\quad(a z)\end{array}$ & $\begin{array}{l}\text { Maximum } \\
\text { amplitude } \\
\text { (g) }\end{array}$ & $\begin{array}{l}\text { Duration } \\
\text { (s) }\end{array}$ \\
\hline \multirow{13}{*}{$\begin{array}{l}11 \text { February } 1988 \\
1525: 55.6 \text { G.m.t. } \\
\text { Southern Calif. } \\
34.080 \mathrm{~N}, 118.050 \mathrm{~W} \\
\text { Magnitude } 4.7 \mathrm{ML} \\
\text { (Continued) }\end{array}$} & $\begin{array}{l}\text { Los Angeles } \\
1100 \text { Wilshire Blvd. } \\
\text { (JCG/USGS) }\end{array}$ & $\begin{array}{r}34.052 \mathrm{~N} \\
118.263 \mathrm{~W}\end{array}$ & $26: 05.0$ & (2) & & & \\
\hline & Basement $3 \mathrm{NE}$ & & & & & (1) & \\
\hline & Basement $3 \mathrm{SE}$ & & & & & (1) & \\
\hline & Basement $4 \mathrm{NW}$ & & & & & (1) & \\
\hline & $\begin{array}{l}\text { Structure Array: } \\
\text { Ch. 1- 12th Floor, } \\
\text { Ch. 2- 12th Floor, } \\
\text { Ch. 3- 12th Floor, } \\
\text { Ch. 4- 13th Floor, } \\
\text { Ch. 5- 13th Floor, } \\
\text { Ch. 6- 13th Floor, } \\
\text { Ch. 7- 32nd Floor, } \\
\text { Ch. 8- 32nd Floor, } \\
\text { Ch. 9- 32nd Floor, } \\
\text { Ch. 10- Ground Floo } \\
\text { Ch. 11- Ground Floo } \\
\text { Ch. 12- Ground Floo }\end{array}$ & $\begin{array}{l}\text { North } \\
\text { North } \\
\text { South } \\
\text { North } \\
\text { North } \\
\text { South } \\
\text { North } \\
\text { North } \\
\text { South } \\
\text { or, North } \\
\text { or, North } \\
\text { or, South }\end{array}$ & & & & $\begin{array}{l}(1) \\
(1) \\
(1) \\
(1) \\
(1) \\
(1) \\
(1) \\
(1) \\
(1) \\
(1) \\
(1) \\
(1)\end{array}$ & \\
\hline & $\begin{array}{l}\text { Orange County } \\
\text { Reservoir, Brea } \\
\text { (MWD) }\end{array}$ & $\begin{array}{r}33.936 \mathrm{~N} \\
117.884 \mathrm{~W}\end{array}$ & (3) & (2) & & & \\
\hline & Abutment & & & & & (1) & \\
\hline & $\begin{array}{l}\text { Brea Dam } \\
\text { Ful lerton } \\
\text { (ACOE) }\end{array}$ & $\begin{array}{r}33.890 \mathrm{~N} \\
117.925 \mathrm{~W}\end{array}$ & (3) & 3.9 & & & \\
\hline & Crest & & & & $\begin{array}{l}130 \\
\text { Up } \\
040\end{array}$ & $\begin{array}{l}.07 \\
.05 \\
.18\end{array}$ & $\begin{array}{l}0.0 \\
0.0 \\
0.7\end{array}$ \\
\hline & Left Abutment & & & & $\begin{array}{l}130 \\
\text { Up } \\
040\end{array}$ & $\begin{array}{l}.05 \\
.04 \\
.07\end{array}$ & $\begin{array}{l}0.0 \\
0.0 \\
0.0\end{array}$ \\
\hline & Downstream & & & & $\begin{array}{l}130 \\
\text { Up } \\
040\end{array}$ & $\begin{array}{l}.05 \\
.04 \\
.09\end{array}$ & $\begin{array}{l}0.0 \\
0.0 \\
0.0\end{array}$ \\
\hline & $\begin{array}{l}\text { Carbon Canyon Dam } \\
\text { Brea } \\
(\mathrm{ACOE})\end{array}$ & $\begin{array}{r}33.914 \mathrm{~N} \\
117.839 \mathrm{~W}\end{array}$ & (3) & 2.5 & & & \\
\hline & Crest & & & & $\begin{array}{l}130 \\
\text { Up } \\
040\end{array}$ & $\begin{array}{l}.05 \\
.02 \\
.05\end{array}$ & $\begin{array}{l}0.0 \\
0.0 \\
0.0\end{array}$ \\
\hline
\end{tabular}


Catalogue of National Cooperative Strong-Hotion Network accelerograph records recovered during 1988--Continued

\begin{tabular}{|c|c|c|c|c|c|c|c|}
\hline Earthquake & $\begin{array}{l}\text { Station name } \\
\text { (owner) }\end{array}$ & $\begin{array}{l}\text { Station } \\
\text { location } \\
\left({ }^{\circ}\right)\end{array}$ & $\begin{array}{c}\text { Trigger } \\
\text { time }\end{array}$ & $\begin{array}{l}\text { S-minus } \\
\text { trigger } \\
(\mathrm{s})\end{array}$ & $\begin{array}{l}\text { Direction } \\
\quad(a z)\end{array}$ & $\begin{array}{l}\text { Maximum } \\
\text { amplitude } \\
(\mathrm{g})\end{array}$ & $\begin{array}{l}\text { Duration } \\
(\mathrm{s})\end{array}$ \\
\hline \multirow{17}{*}{$\begin{array}{l}11 \text { February } 1988 \\
1525: 55.6 \text { G.m.t. } \\
\text { Southern Cal if. } \\
34.080 \mathrm{~N}, 118.050 \mathrm{~W} \\
\text { Magnitude } 4.7 \mathrm{ML} \\
\text { (Cont inued) }\end{array}$} & $\begin{array}{l}\text { Diemer Filter Plant } \\
\text { Yorba Linda } \\
\text { (MwD) }\end{array}$ & $\begin{array}{r}33.913 \mathrm{~N} \\
117.819 \mathrm{~W}\end{array}$ & (3) & 3.5 & & & \\
\hline & Adm in. Bldg. Basement & & & & & (1) & \\
\hline & Reservoir Roof & & & & & (1) & \\
\hline & $\begin{array}{l}\text { Long Beach, CSULB } \\
\text { Human ities BIdg. } \\
\text { Basement (USGS) }\end{array}$ & $\begin{array}{r}33.777 \mathrm{~N} \\
118.112 \mathrm{~W}\end{array}$ & (3) & 2.2 & & (1) & \\
\hline & $\begin{array}{l}\text { Long Beach } \\
\text { VA Hospital } \\
\text { (VA) }\end{array}$ & $\begin{array}{r}33.778 \mathrm{~N} \\
118.118 \mathrm{~W}\end{array}$ & (3) & 3.0 & & & \\
\hline & Basement & & & & & (1) & \\
\hline & 6 th Floor & & & & & (1) & \\
\hline & 11th Floor & & & & & $(1)$ & \\
\hline & $\begin{array}{l}\text { San Antonio Dam } \\
\text { Upland } \\
\text { (ACOE) }\end{array}$ & $\begin{array}{r}34.157 \mathrm{~N} \\
117.676 \mathrm{~W}\end{array}$ & (3) & (2) & & & \\
\hline & Crest & & & & $\begin{array}{l}090 \\
U p \\
360\end{array}$ & $\begin{array}{l}.02 \\
.02 \\
.06\end{array}$ & $\begin{array}{l}0.0 \\
0.0 \\
0.0\end{array}$ \\
\hline & $\begin{array}{l}\text { Prado Dam } \\
\text { Corona } \\
\text { (ACOE) }\end{array}$ & $\begin{array}{r}33.890 \mathrm{~N} \\
117.641 \mathrm{~W}\end{array}$ & (3) & (2) & & & \\
\hline & Crest & & & & & (1) & \\
\hline & Downstream & & & & $\begin{array}{l}090 \\
U p \\
360\end{array}$ & $\begin{array}{l}.07 \\
.02 \\
.08\end{array}$ & $\begin{array}{l}0.0 \\
0.0 \\
0.0\end{array}$ \\
\hline & $\begin{array}{l}\text { Jensen Filter Plant } \\
\text { Balboa Ave. } \\
\text { (MWD) }\end{array}$ & $\begin{array}{r}34.312 \mathrm{~N} \\
118.496 \mathrm{~W}\end{array}$ & (3) & (2) & & & \\
\hline & Administration Bldg. & & & & & (1) & \\
\hline & Generator B $1 \mathrm{dg}$. & & & & & (1) & \\
\hline & Reservoir Roof & & & & & (1) & \\
\hline $\begin{array}{l}20 \text { February } 1988 \\
0447: 04.7 \text { G.m.t. } \\
\text { Hawa } i j \\
19.354 \mathrm{~N}, 155.026 \mathrm{~W} \\
\text { Magnitude } 4.1 \mathrm{ML}\end{array}$ & $\begin{array}{l}\text { Hawa ii National Park } \\
\text { Wahaula Maint. Center } \\
\text { (USGS) }\end{array}$ & $\begin{array}{r}19.329 \mathrm{~N} \\
155.031 \mathrm{~W}\end{array}$ & $47: 05.7^{4}$ & $7 \quad 1.4$ & & (1) & \\
\hline
\end{tabular}


Catalogue of National Cooperative Strong-Motion Network

accelerograph records recovered during 1988--Continued

\begin{tabular}{|c|c|c|c|c|c|c|c|}
\hline Earthquake & $\begin{array}{l}\text { Station name } \\
\text { (owner) }\end{array}$ & $\begin{array}{l}\text { Station } \\
\text { location } \\
\left({ }^{\circ}\right)\end{array}$ & $\underset{\text { Trigger }}{\text { Trime }} \stackrel{s}{t}$ & $\begin{array}{l}\text { S-minus } \\
\text { trigger } \\
(\mathrm{s})\end{array}$ & $\begin{array}{l}\text { Direction } \\
\quad(a z)\end{array}$ & $\begin{array}{l}\text { Maximum } \\
\text { ampl litude } \\
(g)\end{array}$ & $\begin{array}{l}\text { Duration } \\
\text { (s) }\end{array}$ \\
\hline \multirow{3}{*}{$\begin{array}{l}20 \text { February } 1988 \\
0839: 57.5 \mathrm{G} . \mathrm{m} . \mathrm{t} . \\
\text { Central Cal if. } \\
36.803 \mathrm{~N}, 121.302 \mathrm{~W} \\
\text { Magnitude } 5.3 \mathrm{ML}\end{array}$} & $\begin{array}{l}\text { Hollister } \\
\text { Damler Residence } \\
\text { (UCB) }\end{array}$ & $\begin{array}{r}36.807 \mathrm{~N} \\
121.408 \mathrm{~W}\end{array}$ & (3) & 1.6 & $\begin{array}{l}360 \\
\text { Up } \\
270\end{array}$ & $\begin{array}{l}.06 \\
.05 \\
.06\end{array}$ & $\begin{array}{l}0.0 \\
0.0 \\
0.0\end{array}$ \\
\hline & $\begin{array}{l}\text { Hollister } \\
\text { Sago Vault } \\
\text { (UCB) }\end{array}$ & $\begin{array}{r}36.765 \mathrm{~N} \\
121.446 \mathrm{~W}\end{array}$ & $40: 02.8$ & 0.4 & $\begin{array}{l}360 \\
\text { Up } \\
270\end{array}$ & $\begin{array}{l}.06 \\
.02 \\
.05\end{array}$ & $\begin{array}{l}0.0 \\
0.0 \\
0.0\end{array}$ \\
\hline & $\begin{array}{l}\text { Hollister Differential } \\
\text { Array, (Triaxial) } \\
\text { (USGS) }\end{array}$ & $\begin{array}{r}36.888 \mathrm{~N} \\
121.413 \mathrm{~W}\end{array}$ & (3) & 2.9 & $\begin{array}{l}255 \\
\text { Up } \\
165\end{array}$ & $\begin{array}{l}.11 \\
.07 \\
.17\end{array}$ & $\begin{array}{l}0.1 \\
0.0 \\
1 \text { peak }\end{array}$ \\
\hline \multirow{3}{*}{$\begin{array}{l}2 \text { March } 1988 \\
0841: 56.5 \text { G.m.t. } \\
\text { Hawa } i \mathrm{i} \\
19.329 \mathrm{~N}, 155.213 \mathrm{~W} \\
\text { Magnitude } 4.9 \mathrm{MB}\end{array}$} & $\begin{array}{l}\text { Kealakekua, Hawai i } \\
\text { Kona Hospital } \\
\text { (USGS) }\end{array}$ & $\begin{array}{r}19.523 \mathrm{~N} \\
155.879 \mathrm{~W}\end{array}$ & $42: 16.1^{4}$ & (2) & & (1) & \\
\hline & $\begin{array}{l}\text { Pahala, Hawaii } \\
\text { Kau Hospital } \\
\text { (USGS) }\end{array}$ & $\begin{array}{r}19.20 \mathrm{~N} \\
155.47 \mathrm{~W}\end{array}$ & (3) & (2) & & (1) & \\
\hline & $\begin{array}{l}\text { Hawai i National Park } \\
\text { Volcano Observatory } \\
\text { (USGS) }\end{array}$ & $\begin{array}{r}19.423 \mathrm{~N} \\
155.291 \mathrm{~W}\end{array}$ & $42: 05.3^{4}$ & 1.2 & & (1) & \\
\hline \multirow{4}{*}{$\begin{array}{l}6 \text { March } 1988 \\
2235: 38.1 \text { G.m.t. } \\
\text { Gulf of Alaska } \\
56.953 \mathrm{~N}, 143.032 \mathrm{~W} \\
\text { Magnitude } 7.4 \mathrm{ML}\end{array}$} & $\begin{array}{l}\text { Bancas Point } \\
\text { (USGS) }\end{array}$ & $\begin{array}{r}59.953 \mathrm{~N} \\
139.635 \mathrm{~W}\end{array}$ & $38: 26.6^{4}$ & (2) & & (1) & \\
\hline & $\begin{array}{l}\text { Sunshine Point } \\
\text { (USGS) }\end{array}$ & $\begin{array}{r}60.180 \mathrm{~N} \\
142.838 \mathrm{~W}\end{array}$ & $38: 22.9^{4}$ & (2) & & (1) & \\
\hline & $\begin{array}{l}\text { Guyot Hills } \\
\text { (USGS) }\end{array}$ & $\begin{array}{r}60.146 \mathrm{~N} \\
141.472 \mathrm{~W}\end{array}$ & $38: 29.3^{4}$ & (2) & & (1) & \\
\hline & $\begin{array}{l}\text { Yakutat } \\
\text { USGS Building } \\
\text { (USGS) }\end{array}$ & $\begin{array}{r}59.543 \mathrm{~N} \\
139.726 \mathrm{~W}\end{array}$ & (3) & (2) & & (1) & \\
\hline $\begin{array}{l}13 \text { March } 1988 \\
\text { Time incomplete } \\
\text { Hawai } \\
\text { Epicenter and } \\
\text { magnitude unknown }\end{array}$ & $\begin{array}{l}\text { Hawa i i National Park } \\
\text { Wahaula Maint. Center } \\
\text { (USGS) }\end{array}$ & $\begin{array}{r}19.329 \mathrm{~N} \\
155.031 \mathrm{~W}\end{array}$ & $28: 48.5^{4}$ & 1.5 & & (1) & \\
\hline $\begin{array}{l}21 \text { March } 1988 \\
\text { Time incomplete } \\
\text { Central Cal if. } \\
\text { Epicenter and } \\
\text { magnitude unknown }\end{array}$ & $\begin{array}{l}\text { Bear Valley Station } 10 \\
\text { Webb Res idence } \\
\text { (USGS) }\end{array}$ & $\begin{array}{r}36.532 \mathrm{~N} \\
121.143 \mathrm{~W}\end{array}$ & (3) & 1.4 & & (1) & \\
\hline $\begin{array}{l}26 \text { March } 1988 \\
1454: 20.4 \text { G.m.t. } \\
\text { Southern Calif. } \\
34.000 \mathrm{~N}, 118.710 \mathrm{~W} \\
\text { Magnitude } 3.7 \mathrm{ML}\end{array}$ & $\begin{array}{l}\text { Malibu Canyon } \\
\text { Monte Nido Fire Stn. } \\
\text { (USGS) }\end{array}$ & $\begin{array}{r}34.08 \mathrm{~N} \\
118.69 \mathrm{~W}\end{array}$ & $54: 23.2$ & 1.8 & & (1) & \\
\hline
\end{tabular}


Catalogue of Mational Cooperative Strong-Motion Network accelerograph records recovered during 1988--Continued

\begin{tabular}{|c|c|c|c|c|c|c|c|}
\hline Earthquake & $\begin{array}{l}\text { Station name } \\
\text { (owner) }\end{array}$ & $\begin{array}{l}\text { Station } \\
\text { location } \\
\left({ }^{\circ}\right)\end{array}$ & $\begin{array}{l}\text { Trigger } \\
\text { time }\end{array}$ & $\begin{array}{l}\text { S-minus } \\
\text { trigger } \\
(\mathrm{s})\end{array}$ & $\begin{array}{l}\text { Direction } \\
\quad(a z)\end{array}$ & $\begin{array}{l}\text { Maximum } \\
\text { amplitude } \\
\text { (g) }\end{array}$ & $\begin{array}{l}\text { Duration } \\
(\mathrm{s})\end{array}$ \\
\hline \multirow{2}{*}{$\begin{array}{l}28 \text { March } 1988 \\
0333: 40.8 \text { G.m.t. } \\
\text { Hawaij } \\
\text { 19.936N, 156.445W } \\
\text { Magnitude 5.6 MB }\end{array}$} & $\begin{array}{l}\text { Kealakekua, Hawaii } \\
\text { Kona Hospital } \\
\text { (USGS) }\end{array}$ & $\begin{array}{r}19.523 \mathrm{~N} \\
155.879 \mathrm{~W}\end{array}$ & $33: 57.5^{4}$ & (2) & & (1) & \\
\hline & $\begin{array}{l}\text { Wa imea, Hawaii } \\
\text { Fire Station } \\
\text { (USGS) }\end{array}$ & $\begin{array}{r}20.026 \mathrm{~N} \\
155.664 \mathrm{~W}\end{array}$ & $34: 12.6^{4}$ & (2) & & (1) & \\
\hline $\begin{array}{l}4 \text { April } 1988 \\
2045: 48.7 \text { G.m.t. } \\
\text { Central Cal if. } \\
\text { 36.303N, 120.405W } \\
\text { Magnitude } 3.5 \mathrm{ML}\end{array}$ & $\begin{array}{l}\text { Coal inga } \\
0 \text { il City } \\
\text { (USGS) }\end{array}$ & $\begin{array}{r}36.229 \mathrm{~N} \\
120.360 \mathrm{~W}\end{array}$ & $45: 50.6$ & 2.1 & & (1) & \\
\hline $\begin{array}{l}21 \text { April } 1988 \\
2346 \text { G.m.t. } \\
\text { Central Calif. } \\
\text { Epicenter and } \\
\text { magnitude unknown }\end{array}$ & $\begin{array}{l}\text { Chalfant Valley Array } \\
\text { Laws } \\
\text { (USGS) }\end{array}$ & $\begin{array}{r}37.402 \mathrm{~N} \\
118.346 \mathrm{~W}\end{array}$ & $46: 09.4^{4}$ & 0.5 & & (1) & \\
\hline \multirow{2}{*}{$\begin{array}{l}11 \text { Miy } 1988 \\
2314: 17.8 \text { G.m.t. } \\
\text { Hawaij } \\
19.797 \mathrm{~N}, 155.518 \mathrm{~W} \\
\text { Magnitude } 4.2 \mathrm{ML}\end{array}$} & $\begin{array}{l}\text { Honokaa, Hawaii } \\
\text { Police Station } \\
\text { (USGS) }\end{array}$ & $\begin{array}{r}20.080 \mathrm{~N} \\
155.465 \mathrm{~W}\end{array}$ & $14: 37.4^{4}$ & 7 (2) & & (1) & \\
\hline & $\begin{array}{l}\text { Waimea, Hawaii } \\
\text { Fire Station } \\
\text { (USGS) }\end{array}$ & $\begin{array}{r}20.026 \mathrm{~N} \\
155.664 \mathrm{~W}\end{array}$ & $14: 38.5^{4}$ & & $\begin{array}{l}360 \\
\text { Up } \\
270\end{array}$ & $\begin{array}{l}.12 \\
.03 \\
.06\end{array}$ & $\begin{array}{l}0.1 \\
0.0 \\
0.0\end{array}$ \\
\hline $\begin{array}{l}16 \text { May } 1988 \\
1740: 18.5 \text { G.m.t. } \\
\text { Gulf of Alaska } \\
59.378 \mathrm{~N}, 146.338 \mathrm{~W} \\
\text { Magnitude } 3.6 \mathrm{ML}\end{array}$ & $\begin{array}{l}\text { Middleton Is land } \\
\text { FAA TS Bldg. } \\
\text { (USGS) }\end{array}$ & $\begin{array}{r}59.443 \mathrm{~N} \\
146.332 \mathrm{~W}\end{array}$ & $39: 18.8^{4}$ & (2) & (1) & & \\
\hline $\begin{array}{l}17 \text { May } 1988 \\
\text { 1938:37.9 G.m.t. } \\
\text { Southern Cal if. } \\
33.240 \mathrm{~N}, 116.250 \mathrm{~W} \\
\text { Magnitude } 3.8 \mathrm{ML}\end{array}$ & $\begin{array}{l}\text { Borrego Springs } \\
\text { Scripps C1inic } \\
\text { (USGS) }\end{array}$ & $\begin{array}{r}33.210 \mathrm{~N} \\
116.330 \mathrm{~W}\end{array}$ & $38: 42.1$ & (2) & & (1) & \\
\hline \multirow{4}{*}{$\begin{array}{l}28 \text { May } 1988 \\
1808: 55.5 \text { G.m.t. } \\
\text { Eastern Calif. } \\
37.497 \mathrm{~N}, 118.880 \mathrm{~W} \\
\text { Magnitude } 4.1 \mathrm{ML}\end{array}$} & $\begin{array}{l}\text { McGee Creek } \\
\text { Crowley Lake } \\
\text { (USGS) (Triaxia1) }\end{array}$ & $\begin{array}{r}37.550 \mathrm{~N} \\
118.811 \mathrm{~W}\end{array}$ & $08: 58.1$ & (2) & $\begin{array}{l}180 \\
\text { Up } \\
090\end{array}$ & $\begin{array}{l}.06 \\
.05 \\
.06\end{array}$ & $\begin{array}{l}0.0 \\
0.0 \\
0.0\end{array}$ \\
\hline & $\begin{array}{l}\text { MCGee Creek } \\
\text { Crowley Lake } \\
\text { (USGS) (Multi-channel) }\end{array}$ & $\begin{array}{r}37.550 \mathrm{~N} \\
118.811 \mathrm{~W}\end{array}$ & $08: 58.1$ & & & & \\
\hline & $166 \mathrm{~m}$ Downhole & & & (2) & & (1) & \\
\hline & $35 \mathrm{~m}$ Downhole & & & (2) & & (1) & \\
\hline
\end{tabular}


Catalogue of National Cooperative Strong-Motion Network

accelerograph records recovered during 1988--Cont inued

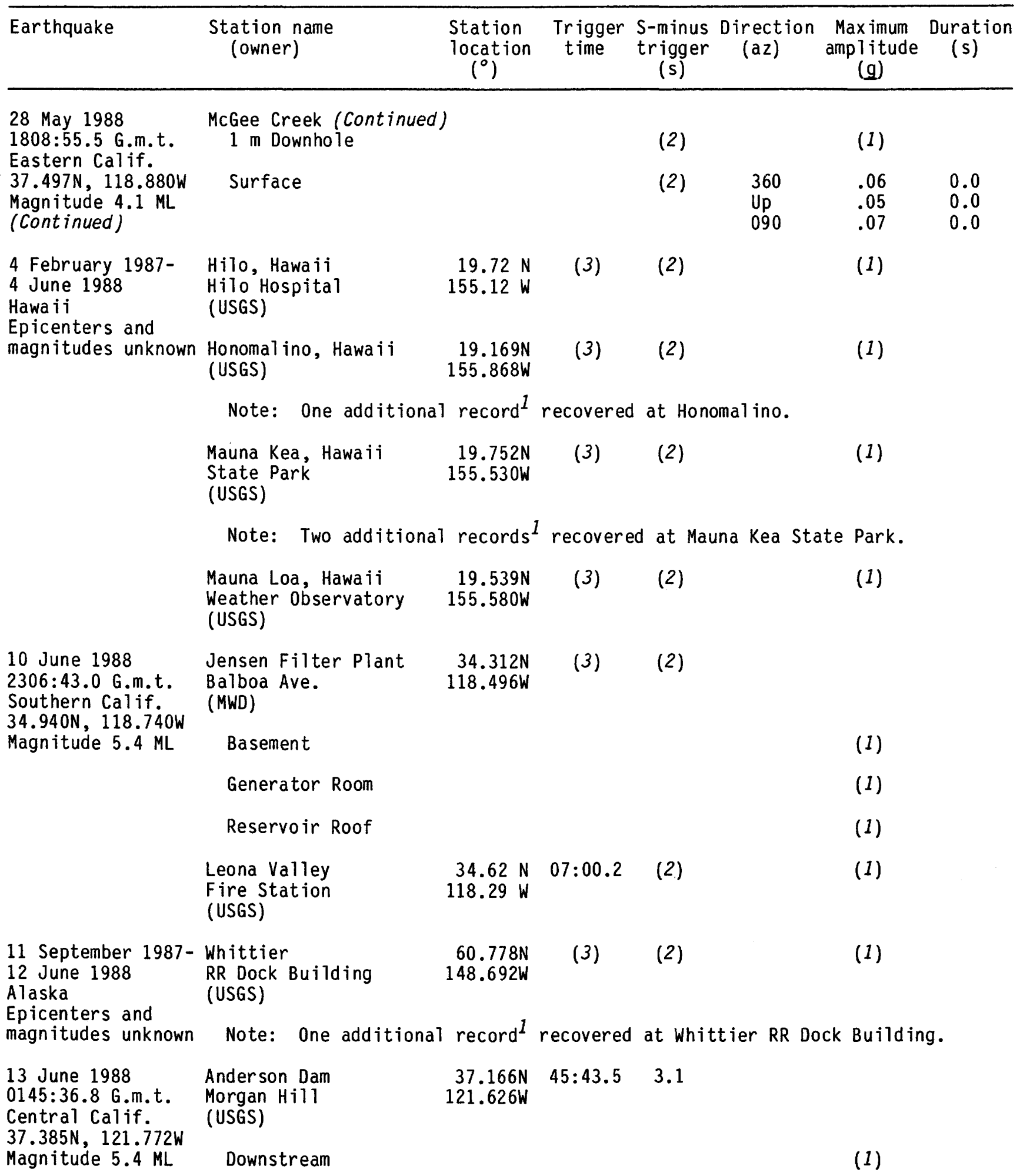


Catalogue of National Cooperative Strong-Motion Network accelerograph records recovered during 1988--Continued

\begin{tabular}{|c|c|c|c|c|c|c|c|}
\hline Earthquake & $\begin{array}{l}\text { Station name } \\
\text { (owner) }\end{array}$ & $\begin{array}{l}\text { Station } \\
\text { location } \\
\left({ }^{\circ}\right)\end{array}$ & $\begin{array}{l}\text { Trigger } \\
\text { time }\end{array}$ & $\begin{array}{l}\text { S-minus } \\
\text { trigger } \\
(\mathrm{s})\end{array}$ & $\begin{array}{l}\text { Direction } \\
\quad(a z)\end{array}$ & $\begin{array}{l}\text { Maximum } \\
\text { ampl itude } \\
(\mathrm{g})\end{array}$ & $\begin{array}{l}\text { Duration } \\
\text { (s) }\end{array}$ \\
\hline \multirow{8}{*}{$\begin{array}{l}13 \text { June } 1988 \\
0145: 36.8 \mathrm{G.m} . \mathrm{t} . \\
\text { Central Cal if. } \\
37.385 \mathrm{~N}, 121.772 \mathrm{~W} \\
\text { Magnitude } 5.4 \mathrm{ML} \\
\text { (Continued) }\end{array}$} & $\begin{array}{l}\text { Livermore VA Hospital } \\
\text { Building } 62 \\
\text { (VA) }\end{array}$ & $\begin{array}{r}37.625 \mathrm{~N} \\
121.762 \mathrm{~W}\end{array}$ & (3) & 3.7 & & & \\
\hline & Basement & & & & & (1) & \\
\hline & Roof (7th Floor) & & & & $\begin{array}{l}125 \\
\text { Up } \\
035\end{array}$ & $\begin{array}{l}.03 \\
.01 \\
.06\end{array}$ & $\begin{array}{l}0.0 \\
0.0 \\
0.0\end{array}$ \\
\hline & $\begin{array}{l}\text { Milpitas } \\
\text { Rivera Street } \\
\text { (USGS) }\end{array}$ & $\begin{array}{r}37.437 \mathrm{~N} \\
121.879 \mathrm{~W}\end{array}$ & (3) & 2.9 & $\begin{array}{l}360 \\
\text { Up } \\
270\end{array}$ & $\begin{array}{l}.09 \\
.02 \\
.07\end{array}$ & $\begin{array}{l}0.0 \\
0.0 \\
0.0\end{array}$ \\
\hline & $\begin{array}{l}\text { Calaveras Array } \\
\text { Sunol Regional Park } \\
\text { (USGS) }\end{array}$ & $\begin{array}{r}37.515 \mathrm{~N} \\
121.830 \mathrm{~W}\end{array}$ & (3) & 2.1 & & (1) & \\
\hline & $\begin{array}{l}\text { Calaveras Array } \\
\text { Calaveras Reserv. So. } \\
\text { (USGS) }\end{array}$ & $\begin{array}{r}37.452 \mathrm{~N} \\
121.807 \mathrm{~W}\end{array}$ & (3) & 1.5 & $\begin{array}{l}180 \\
\text { Up } \\
090\end{array}$ & $\begin{array}{l}.07 \\
.03 \\
.08\end{array}$ & $\begin{array}{l}0.0 \\
0.0 \\
0.0\end{array}$ \\
\hline & $\begin{array}{l}\text { Calaveras Array } \\
\text { Sunol Forest Fire Sta. } \\
\text { (USGS) }\end{array}$ & $\begin{array}{r}37.597 \mathrm{~N} \\
121.880 \mathrm{~W}\end{array}$ & (3) & (2) & $\begin{array}{l}180 \\
\text { Up } \\
090\end{array}$ & $\begin{array}{l}.06 \\
.03 \\
.06\end{array}$ & $\begin{array}{l}0.0 \\
0.0 \\
0.0\end{array}$ \\
\hline & $\begin{array}{l}\text { Fremont, Emerson Ct. } \\
\text { Miss ion San Jose } \\
\text { (USGS) }\end{array}$ & $\begin{array}{r}37.535 \mathrm{~N} \\
121.929 \mathrm{~W}\end{array}$ & (3) & 3.5 & $\begin{array}{l}180 \\
\text { Up } \\
090\end{array}$ & $\begin{array}{l}.06 \\
.05 \\
.10\end{array}$ & $\begin{array}{l}0.0 \\
0.0 \\
1 \text { peak }\end{array}$ \\
\hline $\begin{array}{l}21 \text { June } 1988 \\
0150: 25.1 \text { G.m.t. } \\
\text { Hawaii } \\
19.314 \mathrm{~N}, 155.490 \mathrm{~W} \\
\text { Magnitude } 4.0 \mathrm{MD}\end{array}$ & $\begin{array}{l}\text { Pahala, Hawaii } \\
\text { Kau Hospital } \\
\text { (USGS) }\end{array}$ & $\begin{array}{r}19.20 \mathrm{~N} \\
155.47 \mathrm{~W}\end{array}$ & $50: 25.7^{4}$ & $7 \quad(2)$ & & (1) & \\
\hline \multirow{2}{*}{$\begin{array}{l}26 \text { June } 1988 \\
1504: 58.4 \text { G.m.t. } \\
\text { Southern Calif. } \\
34.140 \mathrm{~N}, 117.710 \mathrm{~W} \\
\text { Magnitude } 4.6 \mathrm{ML}\end{array}$} & $\begin{array}{l}\text { Live Oak Reservoir } \\
\text { LaVerne } \\
\text { (MWD) }\end{array}$ & $\begin{array}{r}34.137 \mathrm{~N} \\
117.753 \mathrm{~W}\end{array}$ & (3) & 0.9 & & & \\
\hline & Abutment & & & & $\begin{array}{l}180 \\
\text { Up } \\
090\end{array}$ & $\begin{array}{l}.23 \\
.08 \\
.12\end{array}$ & $\begin{array}{l}0.6 \\
0.0 \\
0.2\end{array}$ \\
\hline
\end{tabular}


Catalogue of National Cooperative Strong-Hotion Network

accelerograph records recovered during 1988--Cont inued

\begin{tabular}{|c|c|c|c|c|c|c|c|}
\hline Earthquake & $\begin{array}{l}\text { Station name } \\
\text { (owner) }\end{array}$ & $\begin{array}{l}\text { Station } \\
\text { location } \\
\left({ }^{\circ}\right)\end{array}$ & $\begin{array}{l}\text { Trigger } \\
\text { time }\end{array}$ & $\begin{array}{l}\text { S-minus } \\
\text { trigger } \\
(s)\end{array}$ & $\begin{array}{l}\text { Direction } \\
\quad(a z)\end{array}$ & $\begin{array}{l}\text { Maximum } \\
\text { amplitude } \\
\text { (g) }\end{array}$ & $\begin{array}{l}\text { Duration } \\
(\mathrm{s})\end{array}$ \\
\hline \multirow[t]{11}{*}{$\begin{array}{l}26 \text { June } 1988 \\
1504: 58.4 \text { G.m.t. } \\
\text { Southern Calif. } \\
34.140 \mathrm{~N}, 117.710 \mathrm{~W} \\
\text { Magnitude } 4.6 \mathrm{ML} \\
\text { (Continued) }\end{array}$} & \multicolumn{2}{|c|}{$\begin{array}{c}\text { Live Oak Reservoir (Continued) } \\
\text { Structure Array: } \\
\text { Channe1 } 1 \text { - Center Crest } \\
\text { Channel } 2 \text { - Center Crest } \\
\text { Channel } 3 \text { - Center Crest } \\
\text { Channel } 4 \text { - Left Crest } \\
\text { Channel } 5 \text { - Left Crest } \\
\text { Channel } 6 \text { - Left Slope } \\
\text { Channel } 7 \text { - Center Slope } \\
\text { Channel } 8 \text { - Center Slope } \\
\text { Channel } 9 \text { - Center Slope } \\
\text { Channel } 10 \text { - Center Toe } \\
\text { Channel } 11 \text { - Center Toe } \\
\text { Channel } 12 \text { - Center Toe }\end{array}$} & & & $\begin{array}{l}155 \\
\text { Up } \\
245 \\
155 \\
245 \\
245 \\
155 \\
\text { Up } \\
245 \\
155 \\
\text { Up } \\
245\end{array}$ & $\begin{array}{l}.22 \\
.10 \\
.28 \\
.21 \\
.30 \\
.20 \\
.16 \\
.05 \\
.19 \\
.19 \\
.07 \\
.16\end{array}$ & $\begin{array}{c}0.3 \\
1 \text { peak } \\
0.5 \\
0.9 \\
0.6 \\
0.3 \\
0.5 \\
0.0 \\
0.4 \\
0.4 \\
0.0 \\
0.1\end{array}$ \\
\hline & $\begin{array}{l}\text { San Antonio Dam } \\
\text { Upland } \\
\text { (ACOE) }\end{array}$ & $\begin{array}{r}34.157 \mathrm{~N} \\
117.676 \mathrm{~W}\end{array}$ & (3) & 0.3 & & & \\
\hline & Right Abutment & & & & $\begin{array}{l}090 \\
\text { Up } \\
360\end{array}$ & $\begin{array}{l}.19 \\
.17 \\
.13\end{array}$ & $\begin{array}{l}0.5 \\
0.3 \\
0.4\end{array}$ \\
\hline & Crest & & & & $\begin{array}{l}090 \\
\text { Up } \\
360\end{array}$ & $\begin{array}{l}.26 \\
.33 \\
.48\end{array}$ & $\begin{array}{l}0.7 \\
1.7 \\
2.2\end{array}$ \\
\hline & Downstream & & & & $\begin{array}{l}090 \\
\text { Up } \\
360\end{array}$ & $\begin{array}{l}.18 \\
.17 \\
.31\end{array}$ & $\begin{array}{l}0.7 \\
1.5 \\
0.7\end{array}$ \\
\hline & $\begin{array}{l}\text { Weymouth Filter Plant } \\
\text { LaVerne } \\
\text { (MWD) }\end{array}$ & $\begin{array}{r}34.115 \mathrm{~N} \\
117.779 \mathrm{~W}\end{array}$ & (3) & 1.5 & & & \\
\hline & Ground Site & & & & $\begin{array}{l}015 \\
\text { Up } \\
285\end{array}$ & $\begin{array}{l}.12 \\
.08 \\
.06\end{array}$ & $\begin{array}{l}1 \text { peak } \\
0.0 \\
0.0\end{array}$ \\
\hline & Reservoir Roof & & & & $\begin{array}{l}015 \\
\text { Up } \\
285\end{array}$ & $\begin{array}{l}.13 \\
.16 \\
.10\end{array}$ & $\begin{array}{l}1.2 \\
2.0 \\
1 \text { peak }\end{array}$ \\
\hline & $\begin{array}{l}\text { Diemer Filter Plant } \\
\text { Yorba Linda } \\
\text { (MWD) }\end{array}$ & $\begin{array}{r}33.913 \mathrm{~N} \\
117.819 \mathrm{~W}\end{array}$ & (3) & 3.7 & & & \\
\hline & \multicolumn{2}{|l|}{$\begin{array}{l}\text { Administration Bldg. } \\
\text { Basement }\end{array}$} & & & & (1) & \\
\hline & Reservoir Roof & & & & & (1) & \\
\hline
\end{tabular}


Catalogue of Mational Cooperative Strong-Motion Metwork

accelerograph records recovered during 1988--Continued

\begin{tabular}{|c|c|c|c|c|c|c|}
\hline Earthquake & $\begin{array}{l}\text { Station name } \\
\text { (owner) }\end{array}$ & $\begin{array}{l}\text { Station } \\
\text { location } \\
\left({ }^{\circ}\right)\end{array}$ & $\begin{array}{l}\text { Trigger } \\
\text { time }\end{array}$ & $\begin{array}{l}\text { S-minus } \\
\text { trigger } \\
(\mathrm{s})\end{array}$ & $\begin{array}{l}\text { Direction } \\
\quad(a z)\end{array}$ & $\begin{array}{l}\text { Maximum } \\
\text { amplitude } \\
(\mathrm{g})\end{array}$ \\
\hline
\end{tabular}

11 January 1988-

29 June 1988

Central Calif.

Epicenters and

magnitudes unknown Roof (7th Level)
$37.40 \mathrm{~N}$

VA Hospital, Bldg. $1 \quad 122.14 \mathrm{~W}$

(VA)
(3)

(2)

$\begin{array}{ll}.04 & 0.0 \\ .03 & 0.0 \\ .07 & 0.0\end{array}$

Note: One additional record ${ }^{I}$ recovered at Palo Alto VA Hospital, Building 1 Roof.

\begin{tabular}{|c|c|c|c|c|c|c|}
\hline \multirow{3}{*}{$\begin{array}{l}2 \text { July } 1988 \\
\text { 0026:58.1 G.m.t. } \\
\text { Southern Calif. } \\
33.480 \mathrm{~N}, 116.440 \mathrm{~W} \\
\text { Magnitude } 4.0 \mathrm{ML}\end{array}$} & $\begin{array}{l}\text { Anza Array } \\
\text { Tule Canyon } \\
\text { (USGS) }\end{array}$ & $\begin{array}{r}33.47 \mathrm{~N} \\
116.64 \mathrm{~W}\end{array}$ & (3) & 2.1 & \multicolumn{2}{|l|}{ (1) } \\
\hline & $\begin{array}{l}\text { Anza Array } \\
\text { Pine Meadow Ranch } \\
\text { (USGS) }\end{array}$ & $\begin{array}{r}33.578 \mathrm{~N} \\
116.589 \mathrm{~W}\end{array}$ & $27: 04.5$ & (2) & (1) & \\
\hline & $\begin{array}{l}\text { Anza Array } \\
\text { Rar ick Springs } \\
\text { (USGS) }\end{array}$ & $\begin{array}{r}33.568 \mathrm{~N} \\
116.510 \mathrm{~W}\end{array}$ & (3) & (2) & (1) & \\
\hline \multirow{2}{*}{$\begin{array}{l}24 \text { May } 1987- \\
3 \text { July } 1988 \\
\text { Alaska } \\
\text { Epicenters and } \\
\text { magnitudes unknown }\end{array}$} & $\begin{array}{l}\text { Talkeetna } \\
\text { FAA-VOR } \\
\text { (USGS) }\end{array}$ & $\begin{array}{r}62.30 \mathrm{~N} \\
150.10 \mathrm{~W}\end{array}$ & (3) & (2) & \multicolumn{2}{|l|}{ (1) } \\
\hline & Note: Two additional & records ${ }^{1}$ & recovered & \multicolumn{3}{|c|}{ at Talkeetna FAA-VOR. } \\
\hline \multirow{3}{*}{$\begin{array}{l}4 \text { JuTy } 1988 \\
0538: 09.3 \text { G.m.t. } \\
\text { Hawaii } \\
\text { 19.221N, 155.459W } \\
\text { Magnitude } 5.2 \mathrm{ML}\end{array}$} & $\begin{array}{l}\text { Hawa i i National Park } \\
\text { Volcano Observatory } \\
\text { (USGS) }\end{array}$ & $\begin{array}{r}19.423 \mathrm{~N} \\
155.291 \mathrm{~W}\end{array}$ & $38: 21.3^{4}$ & (2) & (1) & \\
\hline & $\begin{array}{l}\text { Pahala, Hawaii } \\
\text { Kau Hospital } \\
\text { (USGS) }\end{array}$ & $\begin{array}{r}19.20 \mathrm{~N} \\
155.47 \mathrm{~W}\end{array}$ & $38: 02.8^{4}$ & $\begin{array}{l}360 \\
\text { Up } \\
270\end{array}$ & $\begin{array}{l}.11 \\
.09 \\
.15\end{array}$ & $\begin{array}{l}0.2 \\
0.0 \\
0.6\end{array}$ \\
\hline & $\begin{array}{l}\text { Wa ioh inu, Hawaii } \\
\text { Kau Baseyard } \\
\text { (USGS) }\end{array}$ & $\begin{array}{r}19.070 \mathrm{~N} \\
155.615 \mathrm{~W}\end{array}$ & (3) & $\begin{array}{l}360 \\
\text { Up } \\
270\end{array}$ & $\begin{array}{l}.06 \\
.06 \\
.16\end{array}$ & $\begin{array}{l}0.0 \\
0.0 \\
0.2\end{array}$ \\
\hline \multirow{3}{*}{$\begin{array}{l}6 \text { July } 1988 \\
\text { 1055:05.5 G.m.t. } \\
\text { Southern Calif. } \\
34.140 \mathrm{~N}, 117.710 \mathrm{~W} \\
\text { Magnitude } 3.8 \mathrm{ML}\end{array}$} & $\begin{array}{l}\text { Diemer Filter Plant } \\
\text { Yorba Linda } \\
\text { (MWD) }\end{array}$ & $\begin{array}{r}33.913 \mathrm{~N} \\
117.819 \mathrm{~W}\end{array}$ & (3) & 3.2 & & \\
\hline & \multicolumn{2}{|l|}{$\begin{array}{l}\text { Administration Bldg. } \\
\text { Basement }\end{array}$} & & & (1) & \\
\hline & \multicolumn{2}{|l|}{ Reservoir Roof } & & & (1) & \\
\hline
\end{tabular}


Catalogue of National Cooperative Strong-Motion Network

accelerograph records recovered during 1988--Cont inued

\begin{tabular}{|c|c|c|c|c|c|c|c|}
\hline Earthquake & $\begin{array}{l}\text { Station name } \\
\text { (owner) }\end{array}$ & $\begin{array}{l}\text { Station } \\
\text { location } \\
\left({ }^{\circ}\right)\end{array}$ & $\begin{array}{l}\text { Trigger } \\
\text { time }\end{array}$ & $\begin{array}{l}\text { S-minus } \\
\text { trigger } \\
(\mathrm{s})\end{array}$ & $\begin{array}{l}\text { Direction } \\
\quad(a z)\end{array}$ & $\begin{array}{l}\text { Maximum } \\
\text { ampl itude } \\
\text { (g) }\end{array}$ & $\begin{array}{l}\text { Duration } \\
\text { (s) }\end{array}$ \\
\hline \multirow{10}{*}{$\begin{array}{l}6 \text { July } 1988 \\
1055: 05.5 \text { G.m.t. } \\
\text { Southern Calif. } \\
34.140 \mathrm{~N}, 117.710 \mathrm{~W} \\
\text { Magnitude } 3.8 \mathrm{ML} \\
\text { (Cont inued) }\end{array}$} & $\begin{array}{l}\text { San Antonio Dam } \\
\text { Upland } \\
\text { (ACOE) }\end{array}$ & $\begin{array}{r}34.157 \mathrm{~N} \\
117.676 \mathrm{~W}\end{array}$ & (3) & (2) & & & \\
\hline & Right Abutment & & & & & (1) & \\
\hline & Crest & & & & $\begin{array}{l}090 \\
\text { Up } \\
360\end{array}$ & $\begin{array}{l}.09 \\
.02 \\
.08\end{array}$ & $\begin{array}{l}0.0 \\
0.0 \\
0.0\end{array}$ \\
\hline & Downstream & & & & $\begin{array}{l}090 \\
\text { Up } \\
360\end{array}$ & $\begin{array}{l}.03 \\
.02 \\
.05\end{array}$ & $\begin{array}{l}0.0 \\
0.0 \\
0.0\end{array}$ \\
\hline & $\begin{array}{l}\text { Live Oak Reservoir } \\
\text { LaVerne } \\
\text { (MWD) }\end{array}$ & $\begin{array}{r}34.137 \mathrm{~N} \\
117.753 \mathrm{~W}\end{array}$ & (3) & (2) & & & \\
\hline & Abutment & & & & $\begin{array}{l}180 \\
\text { Up } \\
090\end{array}$ & $\begin{array}{l}.07 \\
.03 \\
.05\end{array}$ & $\begin{array}{l}0.0 \\
0.0 \\
0.0\end{array}$ \\
\hline & $\begin{array}{l}\text { Structure Array: } \\
\text { Channel } 1 \text { - Center } \\
\text { Channel } 2 \text { - Center } \\
\text { Channel } 3 \text { - Center } \\
\text { Channel } 4 \text { - Left Cr } \\
\text { Channel } 5 \text { - Left Cr } \\
\text { Channel } 6 \text { - Left S1 } \\
\text { Channel } 7 \text { - Center } \\
\text { Channel } 8 \text { - Center } \\
\text { Channel } 9 \text { - Center } \\
\text { Channel } 10 \text { - Center } \\
\text { Channel } 11 \text { - Center } \\
\text { Channel } 12 \text { - Center }\end{array}$ & $\begin{array}{l}\text { Crest } \\
\text { Crest } \\
\text { Crest } \\
\text { rest } \\
\text { rest } \\
\text { lope } \\
\text { Slope } \\
\text { Slope } \\
\text { Slope } \\
r \text { Toe } \\
r \text { Toe } \\
r \text { Toe }\end{array}$ & & & $\begin{array}{l}155 \\
\text { Up } \\
245 \\
155 \\
245 \\
245 \\
155 \\
\text { Up } \\
245 \\
155 \\
\text { Up } \\
245\end{array}$ & $\begin{array}{l}.06 \\
.03 \\
.06 \\
.09 \\
.07 \\
.05 \\
.05 \\
.02 \\
.01 \\
.05 \\
.02 \\
.03\end{array}$ & $\begin{array}{l}0.0 \\
0.0 \\
0.0 \\
0.0 \\
0.0 \\
0.0 \\
0.0 \\
0.0 \\
0.0 \\
0.0 \\
0.0 \\
0.0\end{array}$ \\
\hline & $\begin{array}{l}\text { Weymouth Filter Plant } \\
\text { LaVerne } \\
\text { (MWD) }\end{array}$ & $\begin{array}{r}34.115 \mathrm{~N} \\
117.779 \mathrm{~W}\end{array}$ & (3) & 1.3 & & & \\
\hline & Ground Site & & & & $\begin{array}{l}015 \\
\text { Up } \\
285\end{array}$ & $\begin{array}{l}.05 \\
.03 \\
.04\end{array}$ & $\begin{array}{l}0.0 \\
0.0 \\
0.0\end{array}$ \\
\hline & Reservoir Roof & & & , & $\begin{array}{l}015 \\
\text { Up } \\
285\end{array}$ & $\begin{array}{l}.06 \\
.08 \\
.05\end{array}$ & $\begin{array}{l}0.0 \\
0.0 \\
0.0\end{array}$ \\
\hline
\end{tabular}


Catalogue of National Cooperative Strong-Motion Network accelerograph records recovered during 1988--Cont inued

\begin{tabular}{|c|c|c|c|c|c|c|c|}
\hline Earthquake & $\begin{array}{l}\text { Station name } \\
\text { (owner) }\end{array}$ & $\begin{array}{l}\text { Station } \\
\text { location } \\
\left({ }^{\circ}\right)\end{array}$ & $\begin{array}{l}\text { Trigger } \\
\text { time }\end{array}$ & $\begin{array}{l}\text { S-minus } \\
\text { trigger } \\
(s)\end{array}$ & $\begin{array}{l}\text { Direction } \\
\quad(a z)\end{array}$ & $\begin{array}{l}\text { Maximum } \\
\text { amplitude } \\
\text { (g) }\end{array}$ & $\begin{array}{l}\text { Duration } \\
\text { (s) }\end{array}$ \\
\hline \multirow{5}{*}{$\begin{array}{l}26 \text { July } 1988 \\
0326: 55.9 \mathrm{G.m.t.} \\
\text { Central Calif. } \\
36.558 \mathrm{~N}, 121.175 \mathrm{~W} \\
\text { Magnitude } 4.7 \mathrm{ML}\end{array}$} & $\begin{array}{l}\text { Bear Valley Station } 1 \\
\text { CDF Fire Station } \\
\text { (USGS) }\end{array}$ & $\begin{array}{r}36.573 \mathrm{~N} \\
121.184 \mathrm{~W}\end{array}$ & $26: 56.6$ & 0.9 & $\begin{array}{l}310 \\
\text { Up } \\
220\end{array}$ & $\begin{array}{l}.14 \\
.08 \\
.31\end{array}$ & $\begin{array}{l}1.0 \\
0.0 \\
0.4\end{array}$ \\
\hline & $\begin{array}{l}\text { Bear Valley Station } 7 \\
\text { Pinnacles National } \\
\text { Monument } \\
\text { (USGS) }\end{array}$ & $\begin{array}{r}36.483 \mathrm{~N} \\
121.184 \mathrm{~W}\end{array}$ & $26: 58.7$ & (2) & & (1) & \\
\hline & $\begin{array}{l}\text { Bear Valley Station } 10 \\
\text { Webb Residence } \\
\text { (USGS) }\end{array}$ & $\begin{array}{r}36.532 \mathrm{~N} \\
121.143 \mathrm{~W}\end{array}$ & (3) & 1.3 & $\begin{array}{l}310 \\
\text { Up } \\
220\end{array}$ & $\begin{array}{l}.14 \\
.08 \\
.15\end{array}$ & $\begin{array}{l}0.2 \\
0.0 \\
0.3\end{array}$ \\
\hline & Note: One addition & al record ${ }^{1}$ & recovere & ed at Web & bb Residenc & ce. & \\
\hline & $\begin{array}{l}\text { Bear Valley Station } 14 \\
\text { Upper Butts Ranch } \\
\text { (USGS) }\end{array}$ & $\begin{array}{r}36.569 \mathrm{~N} \\
121.043 \mathrm{~W}\end{array}$ & $27: 01.9$ & (2) & $\begin{array}{l}310 \\
\text { Up } \\
220\end{array}$ & $\begin{array}{l}.04 \\
.03 \\
.06\end{array}$ & $\begin{array}{l}0.0 \\
0.0 \\
0.0\end{array}$ \\
\hline \multirow{3}{*}{$\begin{array}{l}26 \text { July } 1988 \\
0348: 36.0 \text { G.m.t. } \\
\text { Central Calif. } \\
36.573 \mathrm{~N}, 121.182 \mathrm{~W} \\
\text { Magnitude } 3.5 \mathrm{ML}\end{array}$} & $\begin{array}{l}\text { Bear Valley Station } 1 \\
\text { CDF Fire Station } \\
\text { (USGS) }\end{array}$ & $\begin{array}{r}36.573 \mathrm{~N} \\
121.184 \mathrm{~W}\end{array}$ & $48: 36.9$ & 0.8 & & (1) & \\
\hline & $\begin{array}{l}\text { Bear Valley Station } 10 \\
\text { Webb Residence } \\
\text { (USGS) }\end{array}$ & $\begin{array}{r}36.532 \mathrm{~N} \\
121.143 \mathrm{~W}\end{array}$ & $48: 37.8$ & 0.9 & $\begin{array}{l}310 \\
\text { Up } \\
220\end{array}$ & $\begin{array}{l}.04 \\
.03 \\
.05\end{array}$ & $\begin{array}{l}0.0 \\
0.0 \\
0.0\end{array}$ \\
\hline & $\begin{array}{l}\text { Bear Valley Station } 14 \\
\text { Upper Butts Ranch } \\
\text { (USGS) }\end{array}$ & $\begin{array}{r}36.569 \mathrm{~N} \\
121.043 \mathrm{~W}\end{array}$ & $48: 41.5$ & (2) & & (1) & \\
\hline $\begin{array}{l}27 \text { July } 1988 \\
0900 \text { G.m.t. } \\
\text { Hawaii } \\
\text { Epicenter and } \\
\text { magnitude unknown }\end{array}$ & $\begin{array}{l}\text { Pahala, Hawai i } \\
\text { Kau Hospital } \\
\text { (USGS) }\end{array}$ & $\begin{array}{r}19.20 \mathrm{~N} \\
155.47 \mathrm{~W}\end{array}$ & $00: 41.2^{4}$ & $4 \quad(2)$ & & (1) & \\
\hline \multirow{2}{*}{$\begin{array}{l}27 \text { July } 1988 \\
1657: 40.4 \text { G.m.t. } \\
\text { Central Calif. } \\
\text { 36.568N, } 121.180 \mathrm{~W} \\
\text { Magnitude } 3.6 \mathrm{ML}\end{array}$} & $\begin{array}{l}\text { Bear Valley Station } 14 \\
\text { Upper Butts Ranch } \\
\text { (USGS) }\end{array}$ & $\begin{array}{r}36.569 \mathrm{~N} \\
121.043 \mathrm{~W}\end{array}$ & $57: 45.8$ & (2) & & (1) & \\
\hline & $\begin{array}{l}\text { Bear Val ley Station } 10 \\
\text { Webb Residence } \\
\text { (USGS) }\end{array}$ & $\begin{array}{r}36.532 \mathrm{~N} \\
121.143 \mathrm{~W}\end{array}$ & $57: 42.1$ & 1.0 & & (1) & \\
\hline $\begin{array}{l}24 \text { August 1987- } \\
21 \text { August } 1988 \\
\text { Alaska } \\
\text { Epicenter and } \\
\text { magnitude unknown }\end{array}$ & $\begin{array}{l}\text { Cape Yakataga } \\
\text { A irport } \\
\text { (USGS) } \\
\text { Note: Parti }\end{array}$ & $\begin{array}{r}60.08 \mathrm{~N} \\
142.43 \mathrm{~W}\end{array}$ & (3) & 10.5 & $\begin{array}{l}360 \\
\text { Up } \\
270\end{array}$ & $\begin{array}{l}.07 \\
.02 \\
.11\end{array}$ & $\begin{array}{l}0.0 \\
0.0 \\
1 \text { peak }\end{array}$ \\
\hline
\end{tabular}


Catalogue of National Cooperative Strong-Hotion Network

accelerograph records recovered during 1988--Continued

\begin{tabular}{|c|c|c|c|c|c|c|c|}
\hline Earthquake & $\begin{array}{l}\text { Station name } \\
\text { (owner) }\end{array}$ & $\begin{array}{l}\text { Station } \\
\text { location } \\
\left({ }^{\circ}\right)\end{array}$ & $\begin{array}{c}\text { Trigger } \\
\text { time }\end{array}$ & $\begin{array}{l}\text { S-minus } \\
\text { trigger } \\
\quad(s)\end{array}$ & $\begin{array}{l}\text { Direction } \\
\quad(a z)\end{array}$ & $\begin{array}{l}\text { Maximum } \\
\text { amplitude } \\
(\mathrm{g})\end{array}$ & $\begin{array}{l}\text { Duration } \\
\text { (s) }\end{array}$ \\
\hline \multirow{3}{*}{$\begin{array}{l}24 \text { August } 1988 \\
1601: 46.1 \text { G.m.t. } \\
\text { Central Cal if. } \\
36.553 \mathrm{~N}, 121.180 \mathrm{~W} \\
\text { Magnitude } 3.0 \mathrm{ML}\end{array}$} & $\begin{array}{l}\text { Bear Valley Station } 1 \\
\text { COF Fire Station } \\
\text { (USGS) }\end{array}$ & $\begin{array}{r}36.573 \mathrm{~N} \\
121.184 \mathrm{~W}\end{array}$ & $01: 47.0$ & 0.6 & $\begin{array}{l}310 \\
\text { Up } \\
220\end{array}$ & $\begin{array}{l}.03 \\
.02 \\
.06\end{array}$ & $\begin{array}{l}0.0 \\
0.0 \\
0.0\end{array}$ \\
\hline & $\begin{array}{l}\text { Bear Valley Station } 10 \\
\text { Webb Res idence } \\
\text { (USGS) }\end{array}$ & $\begin{array}{r}36.532 \mathrm{~N} \\
121.143 \mathrm{~W}\end{array}$ & $01: 47.2$ & 1.0 & $\begin{array}{l}310 \\
\text { Up } \\
220\end{array}$ & $\begin{array}{l}.08 \\
.03 \\
.07\end{array}$ & $\begin{array}{l}0.0 \\
0.0 \\
0.0\end{array}$ \\
\hline & $\begin{array}{l}\text { Note: One addition } \\
\text { Bear Valley Station } 14 \\
\text { Upper Butts -Ranch } \\
\text { (USGS) }\end{array}$ & $\begin{array}{l}\text { at record }{ }^{1} \\
36.569 \mathrm{~N} \\
121.043 \mathrm{~W}\end{array}$ & $\begin{array}{l}\text { recovered } \\
01: 52.1\end{array}$ & $\begin{array}{l}\text { ed at Bea } \\
\text { (2) }\end{array}$ & ar Valley & $\begin{array}{l}\text { Station } 10 . \\
\text { (1) }\end{array}$ & \\
\hline $\begin{array}{l}30 \text { August } 1988 \\
1228: 25.1 \text { G.m.t. } \\
\text { Eastern Calif. } \\
37.512 \mathrm{~N}, 118.407 \mathrm{~W} \\
\text { Magnitude } 3.5 \mathrm{MD}\end{array}$ & $\begin{array}{l}\text { Chalfant Valley Array } \\
\text { Fire Station } \\
\text { (USGS) }\end{array}$ & $\begin{array}{r}37.53 \mathrm{~N} \\
118.37 \mathrm{~W}\end{array}$ & $28: 27.6^{4}$ & 1.7 & $\begin{array}{l}360 \\
\text { Up } \\
270\end{array}$ & $\begin{array}{l}.09 \\
.03 \\
.06\end{array}$ & $\begin{array}{l}0.0 \\
0.0 \\
0.0\end{array}$ \\
\hline $\begin{array}{l}12 \text { September } 1988 \\
1324: 34.2 \text { G.m.t. } \\
\text { Southern Calif. } \\
33.870 \mathrm{~N}, 118.460 \mathrm{~W} \\
\text { Magnitude } 3.9 \mathrm{ML}\end{array}$ & $\begin{array}{l}\text { Lawndale } \\
15000 \text { Aviation Blvd. } \\
\text { (USGS) }\end{array}$ & $\begin{array}{r}33.895 \mathrm{~N} \\
118.377 \mathrm{~W}\end{array}$ & (3) & 2.3 & & (1) & \\
\hline $\begin{array}{l}14 \text { September } 1988 \\
2121 \text { G.m.t. } \\
\text { Eastern Calif. } \\
\text { Epicenter and } \\
\text { magnitude unknown }\end{array}$ & $\begin{array}{l}\text { Chalfant Valley Array } \\
\text { Fire Station } \\
\text { (USGS) }\end{array}$ & $\begin{array}{r}37.53 \mathrm{~N} \\
118.37 \mathrm{~W}\end{array}$ & (3) & (2) & & (1) & \\
\hline $\begin{array}{l}22 \text { July } 1987- \\
21 \text { September } 1988 \\
\text { Southern Alaska } \\
\text { Epicenter and } \\
\text { magnitude unknown }\end{array}$ & $\begin{array}{l}\text { Thompson Pass } \\
\text { Hiway Maint. Station } \\
\text { (USGS) }\end{array}$ & $\begin{array}{r}61.138 \mathrm{~N} \\
145.741 \mathrm{~W}\end{array}$ & (3) & (2) & $\begin{array}{l}360 \\
\text { Up } \\
270\end{array}$ & $\begin{array}{l}.11 \\
.03 \\
.06\end{array}$ & $\begin{array}{l}0.5 \\
0.0 \\
0.0\end{array}$ \\
\hline \multirow{4}{*}{$\begin{array}{l}3 \text { October } 1988 \\
0406: 42.8 \text { G.m.t. } \\
\text { Hawai } 1 \\
19.777 \mathrm{~N}, 155.329 \mathrm{~W} \\
\text { Magnitude } 4.1 \mathrm{MD}\end{array}$} & $\begin{array}{l}\text { Honokaa, Hawaii } \\
\text { Police Station } \\
\text { (USGS) }\end{array}$ & $\begin{array}{r}20.080 \mathrm{~N} \\
155.465 \mathrm{~W}\end{array}$ & $06: 49.3^{4}$ & 5.1 & $\begin{array}{l}110 \\
\text { Up } \\
020\end{array}$ & $\begin{array}{l}.07 \\
.06 \\
.06\end{array}$ & $\begin{array}{l}0.0 \\
0.0 \\
0.0\end{array}$ \\
\hline & $\begin{array}{l}\text { Laupahoehoe, Hawaii } \\
\text { Post Office } \\
\text { (USGS) }\end{array}$ & $\begin{array}{r}19.987 \mathrm{~N} \\
155.236 \mathrm{~W}\end{array}$ & $06: 38.7^{4}$ & 4.4 & $\begin{array}{l}360 \\
\text { Up } \\
270\end{array}$ & $\begin{array}{l}.05 \\
.02 \\
.03\end{array}$ & $\begin{array}{l}0.0 \\
0.0 \\
0.0\end{array}$ \\
\hline & $\begin{array}{l}\text { Mauna Kea, Hawaii } \\
\text { Summit Observatory } \\
\text { (USGS) }\end{array}$ & $\begin{array}{r}19.826 \mathrm{~N} \\
155.473 \mathrm{~W}\end{array}$ & $07: 26.3^{4}$ & (2) & & (1) & \\
\hline & $\begin{array}{l}\text { Wa imea, Hawa i i } \\
\text { Fire Station } \\
\text { (USGS) }\end{array}$ & $\begin{array}{r}20.026 \mathrm{~N} \\
155.664 \mathrm{~W}\end{array}$ & $05: 59.2^{4}$ & (2) & & (1) & \\
\hline
\end{tabular}


Catalogue of National Cooperative Strong-Motion Network accelerograph records recovered during 1988--Continued

\begin{tabular}{|c|c|c|c|c|c|c|c|}
\hline Earthquake & $\begin{array}{l}\text { Station name } \\
\text { (owner) }\end{array}$ & $\begin{array}{l}\text { Station } \\
\text { location } \\
\left({ }^{\circ}\right)\end{array}$ & $\begin{array}{l}\text { Trigger } \\
\text { time }\end{array}$ & $\begin{array}{l}\text { S-minus } \\
\text { trigger } \\
(\mathrm{s})\end{array}$ & $\begin{array}{l}\text { Direction } \\
\quad(a z)\end{array}$ & $\begin{array}{l}\text { Maximum } \\
\text { amplitude } \\
\text { (g) }\end{array}$ & $\begin{array}{l}\text { Duration } \\
\text { (s) }\end{array}$ \\
\hline $\begin{array}{l}6 \text { May } 1988- \\
13 \text { October } 1988 \\
\text { Eastern Calif. } \\
\text { Epicenter and } \\
\text { magnitude unknown }\end{array}$ & $\begin{array}{l}\text { Chalfant Valley Array } \\
\text { South Hammil Valley } \\
\text { (USGS) }\end{array}$ & $\begin{array}{r}37.62 \mathrm{~N} \\
118.39 \mathrm{~W}\end{array}$ & (3) & 1.5 & & (1) & \\
\hline $\begin{array}{l}15 \text { october } 1988 \\
1952 \text { G.m.t. } \\
\text { Southern Calif. } \\
\text { Epicenter and } \\
\text { magnitude unknown }\end{array}$ & $\begin{array}{l}\text { Salton Sea } \\
\text { Wildlife Refuge } \\
\text { (USGS) }\end{array}$ & $\begin{array}{r}33.18 \mathrm{~N} \\
115.62 \mathrm{~W}\end{array}$ & $52: 06.1$ & 0.4 & & (1) & \\
\hline $\begin{array}{l}19 \text { october } 1988 \\
1608: 23.8 \mathrm{G} . \mathrm{m} . \mathrm{t} . \\
\text { Eastern Calif. } \\
37.203 \mathrm{~N}, 118.452 \mathrm{~W} \\
\text { Magnitude } 4.1 \mathrm{ML}\end{array}$ & $\begin{array}{l}\text { Chalfant Valley Array } \\
\text { Laws, Calif. } \\
\text { (USGS) }\end{array}$ & $\begin{array}{r}37.402 \mathrm{~N} \\
118.346 \mathrm{~W}\end{array}$ & $08: 28.1^{4}$ & $\begin{array}{ll}4 & 3.4\end{array}$ & $\begin{array}{l}360 \\
\text { Up } \\
270\end{array}$ & $\begin{array}{l}.05 \\
.03 \\
.02\end{array}$ & $\begin{array}{l}0.0 \\
0.0 \\
0.0\end{array}$ \\
\hline \multirow[t]{2}{*}{$\begin{array}{l}19 \text { October } 1988 \\
2247: 54.4 \text { G.m.t. } \\
\text { Southern Calif. } \\
33.180 \mathrm{~N}, 115.600 \mathrm{~W} \\
\text { Magnitude } 3.7 \mathrm{ML}\end{array}$} & $\begin{array}{l}\text { Salton Sea } \\
\text { Wildlife Refuge } \\
\text { (USGS) }\end{array}$ & $\begin{array}{r}33.18 \mathrm{~N} \\
115.62 \mathrm{~W}\end{array}$ & {$[48: 42.3]$} & ]$[1.1]$ & $\begin{array}{l}315 \\
\text { Up } \\
225\end{array}$ & $\begin{array}{l}.30 \\
.28 \\
.23 \\
(1)\end{array}$ & $\begin{array}{l}0.8 \\
1.0 \\
0.7\end{array}$ \\
\hline & $\begin{array}{l}\text { Imperial Wildlife } \\
\text { Liquefaction Array } \\
\text { (USGS) }\end{array}$ & $\begin{array}{r}33.097 \mathrm{~N} \\
115.530 \mathrm{~W}\end{array}$ & (3) & 1.3 & & (1) & \\
\hline $\begin{array}{l}19 \text { 0ctober } 1988 \\
2251 \text { G.m.t. } \\
\text { Southern Calif. } \\
\text { Epicenter and } \\
\text { magnitude unknown }\end{array}$ & $\begin{array}{l}\text { Salton Sea } \\
\text { Wildlife Refuge } \\
\text { (USGS) }\end{array}$ & $\begin{array}{r}33.18 \mathrm{~N} \\
115.62 \mathrm{~W}\end{array}$ & $51: 15.35$ & $5 \quad 0.9$ & $\begin{array}{l}315 \\
\text { Up } \\
225\end{array}$ & $\begin{array}{l}.07 \\
.03 \\
.07\end{array}$ & $\begin{array}{l}0.0 \\
0.0 \\
0.0\end{array}$ \\
\hline \multirow{3}{*}{$\begin{array}{l}19 \text { october } 1988 \\
2255: 47.5 \text { G.m.t. } \\
\text { Southern Calif. } \\
33.190 \mathrm{Nn} 115.610 \mathrm{~W} \\
\text { Magnitude } 3.4 \mathrm{ML} \\
22 \text { october } 1988 \\
0238: 18.6 \mathrm{G.m.t} \text {. } \\
\text { Eastern Calif. } \\
37.398 \mathrm{~N}, 118.411 \mathrm{~W} \\
\text { Magnitude } 3.7 \mathrm{MD}\end{array}$} & $\begin{array}{l}\text { Salton Sea } \\
\text { Wildlife Refuge } \\
\text { (USGS) }\end{array}$ & $\begin{array}{r}33.18 \mathrm{~N} \\
115.62 \mathrm{~W}\end{array}$ & $55: 47.7$ & 0.9 & $\begin{array}{l}315 \\
\text { Up } \\
225\end{array}$ & $\begin{array}{l}.05 \\
.15 \\
.07\end{array}$ & $\begin{array}{l}0.0 \\
1 \text { peak } \\
0.0\end{array}$ \\
\hline & Note: One additiona & 1 record 1 & \multicolumn{5}{|c|}{ recorded at Salton Sea Wildlife Refuge. } \\
\hline & $\begin{array}{l}\text { Chalfant Valley Array } \\
\text { Fire Station } \\
\text { (USGS) }\end{array}$ & $\begin{array}{r}37.53 \mathrm{~N} \\
118.37 \mathrm{~W}\end{array}$ & $38: 22.3^{4}$ & $\begin{array}{ll}4 & 2.9\end{array}$ & & (1) & \\
\hline $\begin{array}{l}15 \text { December } 1986- \\
31 \text { October } 1988 \\
\text { Central Calif. } \\
\text { Epicenter and } \\
\text { magnitude unknown }\end{array}$ & $\begin{array}{l}\text { Bear Valley Station } 2 \\
\text { Stone Canyon West } \\
\text { (USGS) }\end{array}$ & $\begin{array}{r}36.636 \mathrm{~N} \\
121.234 \mathrm{~W}\end{array}$ & (3) & (2) & & (1) & \\
\hline
\end{tabular}


Catalogue of National Cooperative Strong-Motion Network accelerograph records recovered during 1988--Continued

\begin{tabular}{|c|c|c|c|c|c|c|c|}
\hline Earthquake & $\begin{array}{l}\text { Station name } \\
\text { (owner) }\end{array}$ & $\begin{array}{l}\text { Station } \\
\text { location } \\
\left({ }^{\circ}\right)\end{array}$ & $\begin{array}{l}\text { Trigger } \\
\text { time }\end{array}$ & $\begin{array}{l}\text { S-minus } \\
\text { trigger } \\
(\mathrm{s})\end{array}$ & $\begin{array}{l}\text { Direction } \\
\quad(a z)\end{array}$ & $\begin{array}{l}\text { Maximum } \\
\text { ampl i tude } \\
(\mathrm{g})\end{array}$ & $\begin{array}{l}\text { Duration } \\
\text { (s) }\end{array}$ \\
\hline $\begin{array}{l}1 \text { November } 1988 \\
0218: 27.2 \text { G.m.t. } \\
\text { Central Calif. } \\
36.555 \mathrm{~N}, 121.177 \mathrm{~W} \\
\text { Magnitude } 2.6 \mathrm{ML}\end{array}$ & $\begin{array}{l}\text { Bear Valley Station } 10 \\
\text { Webb Residence } \\
\text { (USGS) }\end{array}$ & $\begin{array}{r}36.532 \mathrm{~N} \\
121.143 \mathrm{~W}\end{array}$ & $18: 28.3$ & 1.0 & $\begin{array}{l}310 \\
\text { Up } \\
220\end{array}$ & $\begin{array}{l}.04 \\
.02 \\
.07\end{array}$ & $\begin{array}{l}0.0 \\
0.0 \\
0.0\end{array}$ \\
\hline \multirow{7}{*}{$\begin{array}{l}10 \text { November } 1988 \\
0508: 03.0 \mathrm{Gm} . \mathrm{m} . \\
\text { Central Calif. } \\
37.373 \mathrm{~N}, 121.757 \mathrm{~W} \\
\text { Magnitude } 4.8 \mathrm{ML}\end{array}$} & $\begin{array}{l}\text { Calaveras Array } \\
\text { Calaveras Reserv. So. } \\
\text { (USGS) }\end{array}$ & $\begin{array}{r}37.452 \mathrm{~N} \\
121.807 \mathrm{~W}\end{array}$ & (3) & 2.1 & $\begin{array}{l}180 \\
\text { Up } \\
090\end{array}$ & $\begin{array}{l}.06 \\
.03 \\
.07\end{array}$ & $\begin{array}{l}0.0 \\
0.0 \\
0.0\end{array}$ \\
\hline & $\begin{array}{l}\text { Milpitas } \\
\text { Rivera Street } \\
\text { (USGS) }\end{array}$ & $\begin{array}{r}37.437 \mathrm{~N} \\
121.879 \mathrm{~W}\end{array}$ & (3) & 3.4 & & (1) & \\
\hline & $\begin{array}{l}\text { San Jose Interchange } \\
101 / 280 / 680 \\
\text { (USGS) }\end{array}$ & $\begin{array}{r}37.340 \mathrm{~N} \\
121.851 \mathrm{~W}\end{array}$ & (3) & (2) & $\begin{array}{l}322 \\
\text { Up } \\
232\end{array}$ & $\begin{array}{l}.17 \\
.02 \\
.09\end{array}$ & $\begin{array}{l}0.1 \\
0.0 \\
0.0\end{array}$ \\
\hline & $\begin{array}{l}\text { Calaveras Array } \\
\text { Sunol Regional Park } \\
\text { (USGS) }\end{array}$ & $\begin{array}{r}37.515 \mathrm{~N} \\
121.830 \mathrm{~W}\end{array}$ & (3) & 2.6 & $\begin{array}{l}360 \\
\text { Up } \\
270\end{array}$ & $\begin{array}{l}.02 \\
.02 \\
.02\end{array}$ & $\begin{array}{l}0.0 \\
0.0 \\
0.0\end{array}$ \\
\hline & $\begin{array}{l}\text { Sunnyvale, Moffett Fld } \\
\text { Lockheed Way } \\
\text { (USGS) }\end{array}$ & $\begin{array}{r}37.418 \mathrm{~N} \\
122.031 \mathrm{~W}\end{array}$ & (3) & 3.8 & $\begin{array}{l}090 \\
\text { Up } \\
360\end{array}$ & $\begin{array}{l}.03 \\
.02 \\
.03\end{array}$ & $\begin{array}{l}0.0 \\
0.0 \\
0.0\end{array}$ \\
\hline & $\begin{array}{l}\text { Sunnyvale } \\
\text { Colton Ave. } \\
\text { (USGS) }\end{array}$ & $\begin{array}{r}37.402 \mathrm{~N} \\
122.024 \mathrm{~W}\end{array}$ & $08: 08.2$ & 4.0 & $\begin{array}{l}360 \\
\text { Up } \\
270\end{array}$ & $\begin{array}{l}.07 \\
.03 \\
.03\end{array}$ & $\begin{array}{l}0.0 \\
0.0 \\
0.0\end{array}$ \\
\hline & $\begin{array}{l}\text { Fremont, Emerson ct. } \\
\text { Mission San Jose } \\
\text { (USGS) }\end{array}$ & $\begin{array}{r}37.535 \mathrm{~N} \\
121.929 \mathrm{~W}\end{array}$ & (3) & 3.9 & $\begin{array}{l}180 \\
\text { Up } \\
090\end{array}$ & $\begin{array}{l}.02 \\
.02 \\
.02\end{array}$ & $\begin{array}{l}0.0 \\
0.0 \\
0.0\end{array}$ \\
\hline \multirow{4}{*}{$\begin{array}{l}1 \text { March 1988- } \\
11 \text { November } 1988 \\
\text { Southern Calif. } \\
\text { Epicenter and } \\
\text { magnitude unknown }\end{array}$} & $\begin{array}{l}\text { Prado Dam } \\
\text { Corona } \\
\text { (ACOE) }\end{array}$ & $\begin{array}{r}33.890 \mathrm{~N} \\
117.641 \mathrm{~W}\end{array}$ & (3) & 3.9 & & & \\
\hline & Downstream & & & & $\begin{array}{l}090 \\
\text { Up } \\
360\end{array}$ & $\begin{array}{l}.05 \\
.03 \\
.05\end{array}$ & $\begin{array}{l}0.0 \\
0.0 \\
0.0\end{array}$ \\
\hline & $\begin{array}{l}\text { Brea Dam } \\
\text { Fullerton } \\
(\text { ACOE })\end{array}$ & $\begin{array}{r}33.890 \mathrm{~N} \\
117.925 \mathrm{~W}\end{array}$ & (3) & (2) & & & \\
\hline & Crest & & & & & (1) & \\
\hline
\end{tabular}


Catalogue of National Cooperative Strong-Motion Network

accelerograph records recovered during 1988--Continued

\begin{tabular}{|c|c|c|c|c|c|c|c|}
\hline Earthquake & $\begin{array}{l}\text { Station name } \\
\text { (owner) }\end{array}$ & $\begin{array}{l}\text { Station } \\
\text { location } \\
\left({ }^{\circ}\right)\end{array}$ & $\begin{array}{l}\text { Trigger } \\
\text { time }\end{array}$ & $\begin{array}{c}\text { S-minus } \\
\text { trigger } \\
(\mathrm{s})\end{array}$ & $\begin{array}{l}\text { Direction } \\
\quad(a z)\end{array}$ & $\begin{array}{l}\text { Maximum } \\
\text { ampl l itude } \\
\text { (g) }\end{array}$ & $\begin{array}{l}\text { Duration } \\
\text { (s) }\end{array}$ \\
\hline \multirow{2}{*}{$\begin{array}{l}20 \text { November } 1988 \\
0539: 28.6 \text { G.m.t. } \\
\text { Southern Calif. } \\
33.510 \mathrm{~N}, 118.070 \mathrm{~W} \\
\text { Magnitude } 4.5 \mathrm{ML}\end{array}$} & $\begin{array}{l}\text { Newport Beach } \\
840 \text { Newport Center } \\
\text { (USGS) }\end{array}$ & $\begin{array}{r}33.618 \mathrm{~N} \\
\text { Dr. } 117.878 \mathrm{~W}\end{array}$ & (3) & 3.3 & & & \\
\hline & $\begin{array}{l}\text { Structure Array } \\
\text { Ch. 1- Tower 2, } \\
\text { Ch. 2- Tower 2, } \\
\text { Ch. 3- Tower 2, } \\
\text { Ch. 4- Tower 2, } \\
\text { Ch. 5- Tower 2, } \\
\text { Ch. 6- Tower 2, } \\
\text { Ch. 7- Tower 2, } \\
\text { Ch. 8- Tower 2, } \\
\text { Ch. 9- Tower 2, } \\
\text { Ch. 10- Tower } 1 \\
\text { Ch. 11- Tower } 1 \\
\text { Ch. 12- Tower } 1\end{array}$ & 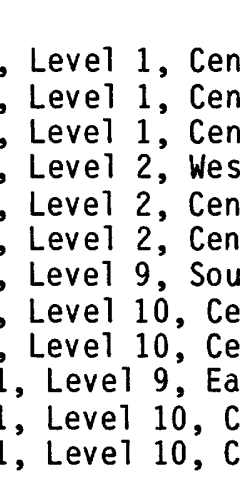 & $\begin{array}{l}\text { ter } \\
\text { ter } \\
\text { ter } \\
t \\
\text { ter } \\
\text { ter } \\
\text { th } \\
\text { nter } \\
\text { nter } \\
\text { st } \\
\text { enter } \\
\text { enter }\end{array}$ & & & $\begin{array}{c}(1) \\
(1) \\
(1) \\
\text { Inoperative } \\
(1) \\
(1) \\
(1) \\
\text { Inoperative } \\
\text { Inoperative } \\
(1) \\
(1) \\
(1)\end{array}$ & \\
\hline
\end{tabular}

1 March 1988-

29 November 1988

Southern Cal if.

Epicenter and

magnitude unknown

Carbon Canyon Dam

Brea

( $A C O E)$

Crest

$33.914 \mathrm{~N}$

$117.839 \mathrm{~W}$

(3)

(2)

11 September 1985- Los Angeles

2 December 1988

Southern Calif.

Epicenters and

magnitudes unknown Roof (8th Level)
$34.098 \mathrm{~N}$
$1526 \mathrm{~N}$. Edgemont St. $\quad 118.294 \mathrm{~W}$ (OWNR)

(3)

(2)

$\begin{array}{lll}090 & .42 & 6.6 \\ \text { Up } & .21 & 6.1 \\ 360 & .27 & 8.1 \\ 090 & .11 & 1 \text { peak } \\ \text { Up } & .05 & 0.0 \\ 360 & .06 & 0.0 \\ & & \\ 090 & .28 & 0.3 \\ \text { Up } & .09 & 0.0 \\ 360 & .06 & 0.0 \\ 090 & .07 & 0.0 \\ \text { Up } & .04 & 0.0 \\ 360 & .03 & 0.0 \\ 090 & .38 & 3.7 \\ \text { Up } & .19 & 0.6 \\ 360 & .15 & 0.3\end{array}$

Note: Three additional records ${ }^{1}$ recovered at 1526 N. Edgemont Street, Roof. 
Catalogue of National Cooperative Strong-Motion Network

accelerograph records recovered during 1988--Continued

\begin{tabular}{|c|c|c|c|c|c|c|c|}
\hline Earthquake & $\begin{array}{l}\text { Station name } \\
\text { (owner) }\end{array}$ & $\begin{array}{l}\text { Station } \\
\text { location } \\
\left({ }^{\circ}\right)\end{array}$ & $\begin{array}{l}\text { Trigger } \\
\text { time }\end{array}$ & $\begin{array}{c}\text { S-minus } \\
\text { trigger } \\
(\mathrm{s})\end{array}$ & $\begin{array}{l}\text { Direction } \\
\quad(a z)\end{array}$ & $\begin{array}{l}\text { Maximum } \\
\text { amplitude } \\
\text { (g) }\end{array}$ & $\begin{array}{l}\text { Duration } \\
\text { (s) }\end{array}$ \\
\hline \multirow{3}{*}{$\begin{array}{l}19 \text { February } 1987- \\
3 \text { December } 1988 \\
\text { Southern Cal if. } \\
\text { Epicenters and } \\
\text { magnitudes unknown }\end{array}$} & $\begin{array}{l}\text { Los Angeles, } 2055 \\
\text { Avenue of the Stars } \\
\text { (OWNR) }\end{array}$ & $\begin{array}{r}34.056 \mathrm{~N} \\
118.413 \mathrm{~W}\end{array}$ & (3) & (2) & & & \\
\hline & Roof (31st level) & & & & $\begin{array}{l}320 \\
\text { Up } \\
230\end{array}$ & $\begin{array}{l}.07 \\
.13 \\
.08\end{array}$ & $\begin{array}{c}0.0 \\
1 \text { peak } \\
0.0\end{array}$ \\
\hline & & & & & $\begin{array}{l}320 \\
\text { Up } \\
230\end{array}$ & $\begin{array}{l}.06 \\
.03 \\
.06\end{array}$ & $\begin{array}{l}0.0 \\
0.0 \\
0.0\end{array}$ \\
\hline
\end{tabular}

Note: Eight additional records ${ }^{1}$ recovered at 2055 Avenue of the Stars, 31 st Level.

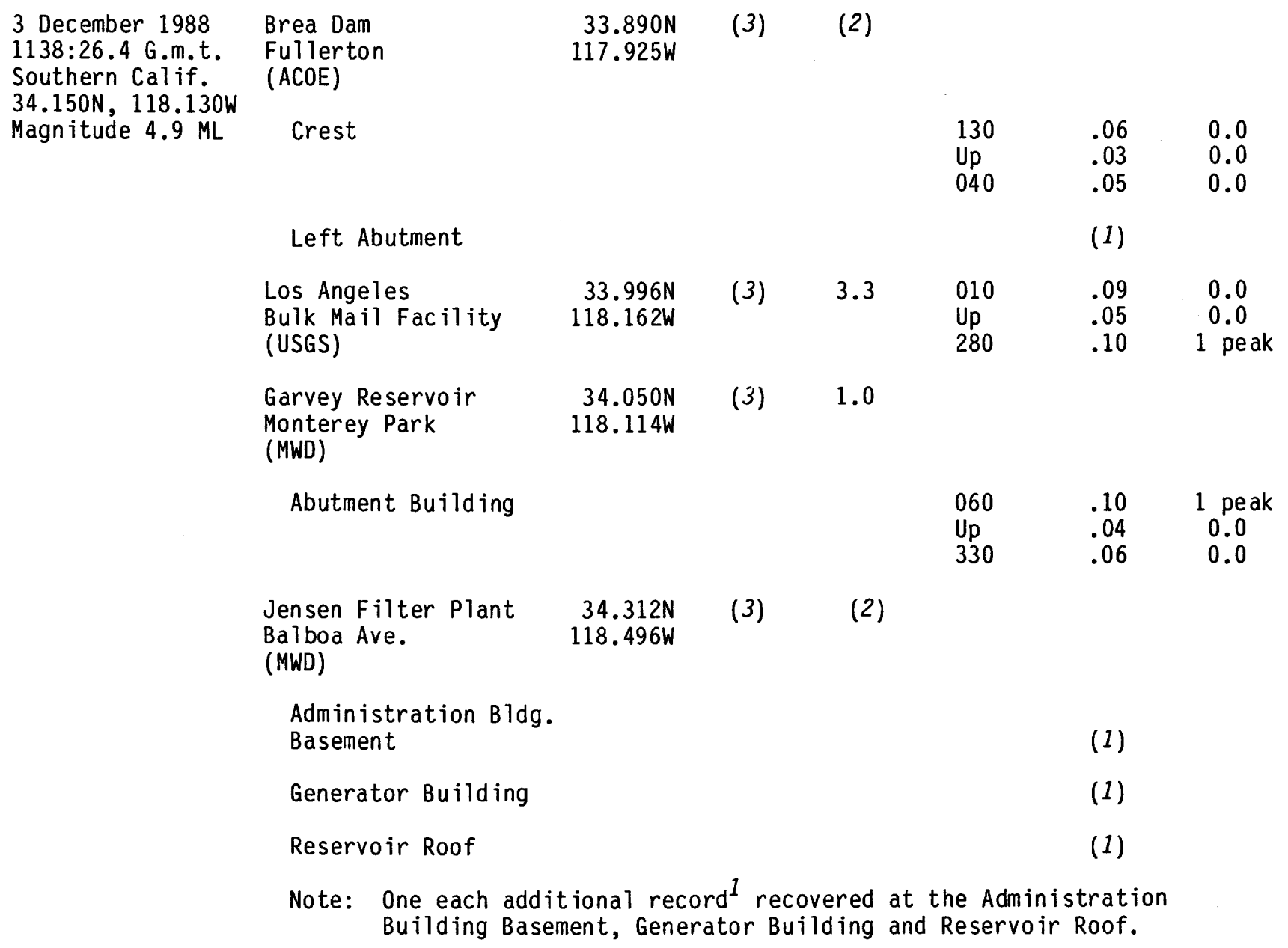


Catalogue of National Cooperative Strong-Motion Network accelerograph records recovered during 1988--Continued

\begin{tabular}{|c|c|c|c|c|c|c|c|}
\hline Earthquake & $\begin{array}{l}\text { Station name } \\
\text { (owner) }\end{array}$ & $\begin{array}{l}\text { Station } \\
\text { location } \\
\left({ }^{\circ}\right)\end{array}$ & $\begin{array}{l}\text { Trigger } \\
\text { time }\end{array}$ & $\begin{array}{l}\text { S-minus } \\
\text { trigger } \\
(\mathrm{s})\end{array}$ & $\begin{array}{l}\text { Direction } \\
\quad(a z)\end{array}$ & $\begin{array}{l}\text { Maximum } \\
\text { amplitude } \\
\text { (g) }\end{array}$ & $\begin{array}{l}\text { Duration } \\
\text { (s) }\end{array}$ \\
\hline \multirow{16}{*}{$\begin{array}{l}3 \text { December } 1988 \\
1138: 26.4 \mathrm{G} . \mathrm{m} . \mathrm{t} . \\
\text { Southern Calif. } \\
34.150 \mathrm{~N}, 118.130 \mathrm{~W} \\
\text { Magnitude } 4.9 \mathrm{ML} \\
\text { (Cont inued) }\end{array}$} & $\begin{array}{l}\text { Long Beach } \\
\text { VA Hospital } \\
\text { (VA) }\end{array}$ & $\begin{array}{r}33.778 \mathrm{~N} \\
118.118 \mathrm{~W}\end{array}$ & (3) & (2) & & & \\
\hline & Basement & & & & & (1) & \\
\hline & 6th Floor & & & & & (1) & \\
\hline & 11th Floor & & & & & (1) & \\
\hline & $\begin{array}{l}\text { Los Angeles, } 2055 \\
\text { Avenue of the Stars } \\
\text { (OWNR) }\end{array}$ & $\begin{array}{r}34.056 \mathrm{~N} \\
118.413 \mathrm{~W}\end{array}$ & (3) & (2) & & & \\
\hline & 31st level & & & & & (1) & \\
\hline & $\begin{array}{l}\text { Los Angeles } \\
\text { Griffith Park Observ. } \\
\text { (USGS) }\end{array}$ & $\begin{array}{r}34.118 \mathrm{~N} \\
118.299 \mathrm{~W}\end{array}$ & (3) & 2.4 & $\begin{array}{l}360 \\
\text { Up } \\
270\end{array}$ & $\begin{array}{l}.04 \\
.05 \\
.08\end{array}$ & $\begin{array}{l}0.0 \\
0.0 \\
0.0\end{array}$ \\
\hline & $\begin{array}{l}\text { Los Angeles } \\
1526 \mathrm{~N} \text {. Edgemont St. } \\
\text { (OWNR) }\end{array}$ & $\begin{array}{r}34.098 \mathrm{~N} \\
118.294 \mathrm{~W}\end{array}$ & (3) & (2) & & & \\
\hline & 8th Level & & & & & (1) & \\
\hline & $\begin{array}{l}\text { Los Angeles, } 2005 \\
\text { N. Highland Blvd. } \\
\text { (OWNR) }\end{array}$ & $\begin{array}{r}34.106 \mathrm{~N} \\
118.336 \mathrm{~W}\end{array}$ & (3) & (2) & & & \\
\hline & 8th Level & & & & & (1) & \\
\hline & $\begin{array}{l}\text { Los Angeles } \\
4407 \text { Jasper Street } \\
\text { (USGS) }\end{array}$ & $\begin{array}{r}34.081 \mathrm{~N} \\
118.188 \mathrm{~W}\end{array}$ & (3) & 1.4 & $\begin{array}{l}130 \\
\text { Up } \\
040\end{array}$ & $\begin{array}{l}.11 \\
.06 \\
.12\end{array}$ & $\begin{array}{l}0.8 \\
0.0 \\
0.2\end{array}$ \\
\hline & $\begin{array}{l}\text { Los Angeles } \\
1111 \text { Sunset Blvd. } \\
\text { (MWD) }\end{array}$ & $\begin{array}{r}34.067 \mathrm{~N} \\
118.248 \mathrm{~W}\end{array}$ & (3) & 1.3 & & & \\
\hline & Basement & & & & $\begin{array}{l}348 \\
\text { Up } \\
258\end{array}$ & $\begin{array}{l}.08 \\
.04 \\
.12\end{array}$ & $\begin{array}{l}0.0 \\
0.0 \\
1 \text { peak }\end{array}$ \\
\hline & 4th Floor & & & & $\begin{array}{l}348 \\
\text { Up } \\
258\end{array}$ & $\begin{array}{l}.08 \\
.04 \\
.09\end{array}$ & $\begin{array}{l}0.0 \\
0.0 \\
0.0\end{array}$ \\
\hline & Roof Level (8th) & & & & $\begin{array}{l}348 \\
\text { Up } \\
258\end{array}$ & $\begin{array}{l}.04 \\
.19 \\
.03\end{array}$ & $\begin{array}{l}0.0 \\
0.9 \\
0.0\end{array}$ \\
\hline
\end{tabular}


Catalogue of National Cooperative Strong-Motion Network

accelerograph records recovered during 1988--Continued

\begin{tabular}{|c|c|c|c|c|c|c|c|}
\hline Earthquake & $\begin{array}{l}\text { Station name } \\
\text { (owner) }\end{array}$ & $\begin{array}{l}\text { Station } \\
\text { location } \\
\left({ }^{\circ}\right)\end{array}$ & $\begin{array}{l}\text { Trigger } \\
\text { time }\end{array}$ & $\begin{array}{l}\text { S-minus } \\
\text { trigger } \\
(\mathrm{s})\end{array}$ & $\begin{array}{l}\text { Direction } \\
\quad(a z)\end{array}$ & $\begin{array}{l}\text { Maximum } \\
\text { amplitude } \\
\text { (g) }\end{array}$ & $\begin{array}{l}\text { Duration } \\
\text { (s) }\end{array}$ \\
\hline \multirow[t]{12}{*}{$\begin{array}{l}3 \text { December } 1988 \\
1138: 26.4 \text { G.m.t. } \\
\text { Southern Calif. } \\
34.150 \mathrm{~N}, 118.130 \mathrm{~W} \\
\text { Magnitude } 4.9 \mathrm{ML} \\
\text { (Cont inued) }\end{array}$} & $\begin{array}{l}\text { Los Angeles } \\
\text { Wadsworth VA Hospital } \\
\text { (USGS) } \\
\quad \text { North ground Site }\end{array}$ & $\begin{array}{r}34.054 \mathrm{~N} \\
118.453 \mathrm{~W}\end{array}$ & $38: 38.7$ & (2) & & (1) & \\
\hline & $\begin{array}{l}\text { Los Angeles } \\
1100 \text { Wilshire Blvd. } \\
\text { (JCG/USGS) }\end{array}$ & $\begin{array}{r}34.052 \mathrm{~N} \\
118.263 \mathrm{~W}\end{array}$ & $38: 32.9$ & 1.5 & & & \\
\hline & Basement $3 \mathrm{NE}$ & & & & $\begin{array}{l}298 \\
\text { Up } \\
208\end{array}$ & $\begin{array}{l}.09 \\
.05 \\
.05\end{array}$ & $\begin{array}{l}0.0 \\
0.0 \\
0.0\end{array}$ \\
\hline & Basement $4 \mathrm{NW}$ & & & & $\begin{array}{l}298 \\
\text { Up } \\
208\end{array}$ & $\begin{array}{l}.08 \\
.03 \\
.06\end{array}$ & $\begin{array}{l}0.0 \\
0.0 \\
0.0\end{array}$ \\
\hline & Basement $3 \mathrm{SE}$ & & & & $\begin{array}{l}298 \\
\text { Up } \\
208\end{array}$ & $\begin{array}{l}.08 \\
.04 \\
.07\end{array}$ & $\begin{array}{l}0.0 \\
0.0 \\
0.0\end{array}$ \\
\hline & $\begin{array}{l}\text { Structure Array: } \\
\text { Ch. 1- 12th Floor, } \\
\text { Ch. 2- 12th Floor, } \\
\text { Ch. 3- 12th Floor, } \\
\text { Ch. 4- 13th Floor, } \\
\text { Ch. 5- 13th Floor, } \\
\text { Ch. 6- 13th Floor, } \\
\text { Ch. 7- 32nd Floor, } \\
\text { Ch. 8- 32nd Floor, } \\
\text { Ch. 9-32nd Floor, } \\
\text { Ch. 10- Ground Flo } \\
\text { Ch. 11- Ground Flo } \\
\text { Ch. 12- Ground Flo }\end{array}$ & $\begin{array}{l}\text {, North } \\
\text {, North } \\
\text {, South } \\
\text {, North } \\
\text {, North } \\
\text {, South } \\
\text {, North } \\
\text {, North } \\
\text {, South } \\
\text { oor, North } \\
\text { oor, North } \\
\text { oor, South }\end{array}$ & & & $\begin{array}{l}298 \\
208 \\
208 \\
298 \\
208 \\
208 \\
298 \\
208 \\
208 \\
298 \\
208 \\
208\end{array}$ & $\begin{array}{l}.04 \\
.06 \\
.07 \\
.06 \\
.11 \\
.07 \\
.04 \\
.09 \\
.03 \\
.06 \\
.09 \\
.07\end{array}$ & $\begin{array}{l}0.0 \\
0.0 \\
0.0 \\
0.0 \\
1 \text { peak } \\
0.0 \\
0.0 \\
0.0 \\
0.0 \\
0.0 \\
0.0 \\
0.0\end{array}$ \\
\hline & $\begin{array}{l}\text { Lytle Creek } \\
\text { Mann Residence } \\
\text { (USGS) }\end{array}$ & $\begin{array}{r}34.26 \mathrm{~N} \\
117.50 \mathrm{~W}\end{array}$ & $38: 54.3$ & (2) & & (1) & \\
\hline & $\begin{array}{l}\text { Norwalk } \\
12400 \text { Imperial Highway } \\
\text { (USGS/BECH) }\end{array}$ & $\begin{array}{r}33.916 \mathrm{~N} \\
118.067 \mathrm{~W}\end{array}$ & (3) & 0.9 & & & \\
\hline & Basement & & & & & (1) & \\
\hline & 4th Floor & & & & & (1) & \\
\hline & North Ground Site & & & & & (1) & \\
\hline & South Ground Site & & & & & (1) & \\
\hline
\end{tabular}


Catalogue of National Cooperative Strong-Motion Network accelerograph records recovered during 1988--Continued

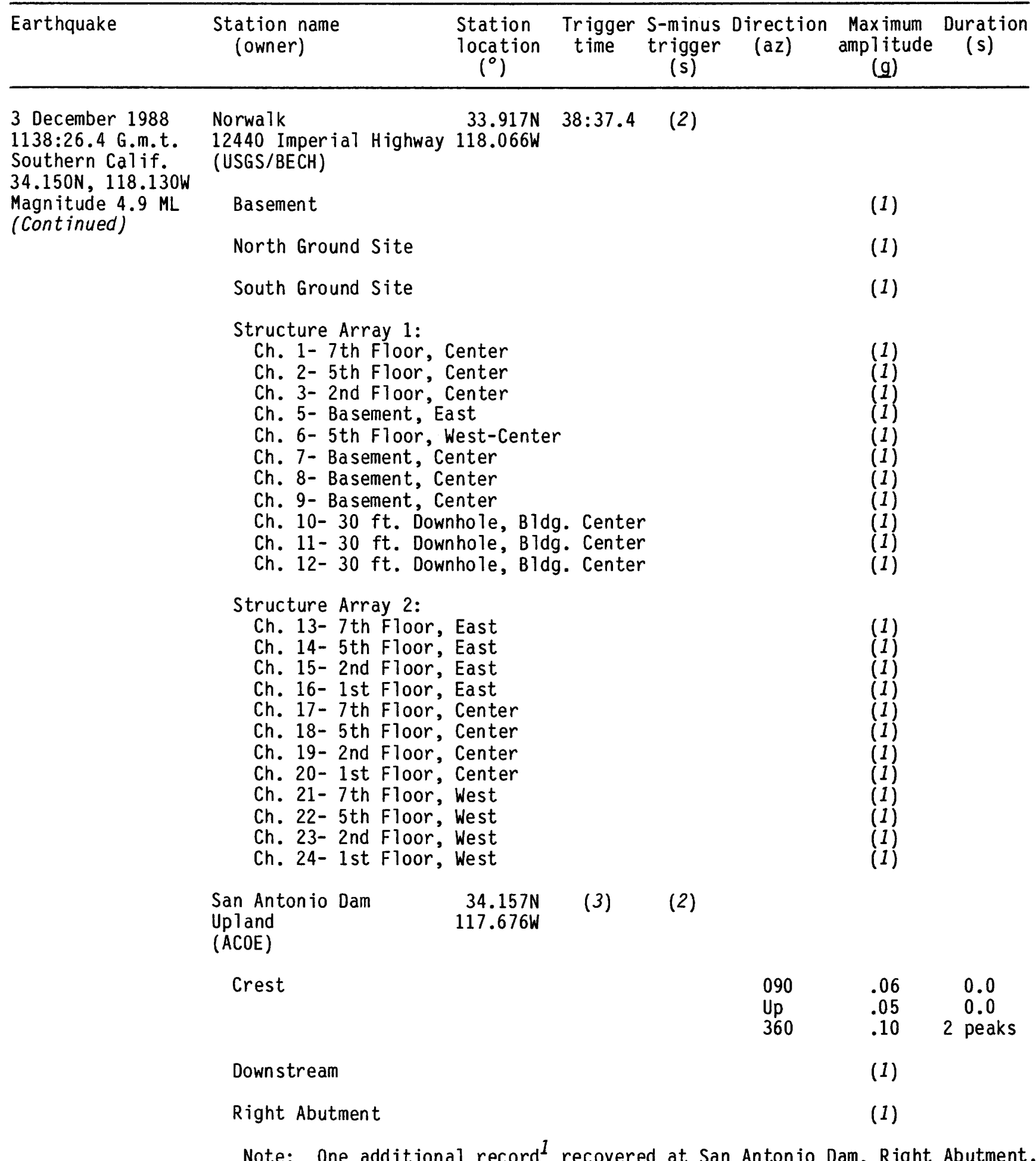

Note: One additional record ${ }^{1}$ recovered at San Antonio Dam, Right Abutment. 
Catalogue of National Cooperative Strong-Motion Network

accelerograph records recovered during 1988--Continued

\begin{tabular}{|c|c|c|c|c|c|c|c|}
\hline Earthquake & $\begin{array}{l}\text { Station name } \\
\text { (owner) }\end{array}$ & $\begin{array}{l}\text { Station } \\
\text { location } \\
\left({ }^{\circ}\right)\end{array}$ & $\begin{array}{l}\text { Trigger } \\
\text { time }\end{array}$ & $\begin{array}{l}\text { S-minus } \\
\text { trigger } \\
\quad(\mathrm{s})\end{array}$ & $\begin{array}{l}\text { Direction } \\
\quad(a z)\end{array}$ & $\begin{array}{l}\text { Max imum } \\
\text { ampl itude } \\
\text { (g) }\end{array}$ & $\begin{array}{l}\text { Duration } \\
\text { (s) }\end{array}$ \\
\hline \multirow{15}{*}{$\begin{array}{l}3 \text { December } 1988 \\
1138: 26.4 \text { G.m.t. } \\
\text { Southern Calif. } \\
34.150 \mathrm{~N}, 118.130 \mathrm{~W} \\
\text { Magnitude } 4.9 \mathrm{ML} \\
\text { (Continued) }\end{array}$} & $\begin{array}{l}\text { Weymouth Filter Plant } \\
\text { LaVerne } \\
\text { (MWD) }\end{array}$ & $\begin{array}{r}34.115 \mathrm{~N} \\
117.779 \mathrm{~W}\end{array}$ & (3) & (2) & & 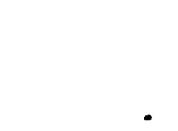 & \\
\hline & Tank Top & & & & & (1) & \\
\hline & Ground Site & & & & & (1) & \\
\hline & $\begin{array}{l}\text { Whittier } \\
7215 \text { Bright Avenue } \\
\text { (USGS/CLA) }\end{array}$ & $\begin{array}{r}33.976 \mathrm{~N} \\
118.036 \mathrm{~W}\end{array}$ & (3) & 0.2 & & & \\
\hline & Basement & & & & $\begin{array}{l}180 \\
\text { Up } \\
090\end{array}$ & $\begin{array}{l}.08 \\
.04 \\
.06\end{array}$ & $\begin{array}{l}0.0 \\
0.0 \\
0.0\end{array}$ \\
\hline & 5th Floor & & & & $\begin{array}{l}180 \\
\text { Up } \\
090\end{array}$ & $\begin{array}{l}.10 \\
.05 \\
.06\end{array}$ & $\begin{array}{l}1 \text { peak } \\
0.0 \\
0.0\end{array}$ \\
\hline & 10th Floor & & & & $\begin{array}{l}180 \\
\text { Up } \\
090\end{array}$ & $\begin{array}{l}.06 \\
.06 \\
.08\end{array}$ & $\begin{array}{l}0.0 \\
0.0 \\
0.0\end{array}$ \\
\hline & $\begin{array}{l}\text { Whittier Narrows Dam } \\
\text { PiCO Rivera } \\
\text { (ACOE) }\end{array}$ & & (3) & (2) & & & \\
\hline & Crest & $\begin{array}{r}34.020 \mathrm{~N} \\
118.053 \mathrm{~W}\end{array}$ & & & $\begin{array}{l}033 \\
U p \\
303\end{array}$ & $\begin{array}{l}.07 \\
.03 \\
.06\end{array}$ & $\begin{array}{l}0.0 \\
0.0 \\
0.0\end{array}$ \\
\hline & Upstream (Baseyard) & $\begin{array}{r}34.031 \mathrm{~N} \\
118.054 \mathrm{~W}\end{array}$ & & & $\begin{array}{l}152 \\
\text { Up } \\
062\end{array}$ & $\begin{array}{l}.07 \\
.03 \\
.06\end{array}$ & $\begin{array}{l}0.0 \\
0.0 \\
0.0\end{array}$ \\
\hline & $\begin{array}{l}\text { Sepulveda Canyon } \\
\text { Control Facility } \\
\text { (MWD) }\end{array}$ & $\begin{array}{r}34.097 \mathrm{~N} \\
118.478 \mathrm{~W}\end{array}$ & (3) & 4.0 & $\begin{array}{l}166 \\
\text { Up } \\
076\end{array}$ & $\begin{array}{l}.02 \\
.02 \\
.08\end{array}$ & $\begin{array}{l}0.0 \\
0.0 \\
0.0\end{array}$ \\
\hline & $\begin{array}{l}\text { Sepulveda Dam } \\
\text { San Fernando Val ley } \\
(A C O E)\end{array}$ & $\begin{array}{r}34.167 \mathrm{~N} \\
118.469 \mathrm{~W}\end{array}$ & (3) & 4.1 & & & \\
\hline & Crest & & & & & (1) & \\
\hline & Downstream & & & & & $(1)$ & \\
\hline & $\begin{array}{l}\text { Sepulveda VA Hospital } \\
\text { Building } \# 40 \\
\text { (VA) }\end{array}$ & $\begin{array}{r}34.249 \mathrm{~N} \\
118.475 \mathrm{~W}\end{array}$ & (3) & 5.2 & $\begin{array}{l}360 \\
\text { Up } \\
270\end{array}$ & $\begin{array}{l}.07 \\
.04 \\
.04\end{array}$ & $\begin{array}{l}0.0 \\
0.0 \\
0.0\end{array}$ \\
\hline
\end{tabular}


Catalogue of National Cooperative Strong-Motion Network accelerograph records recovered during 1988--Cont inued

\begin{tabular}{|c|c|c|c|c|c|c|c|}
\hline Earthquake & $\begin{array}{l}\text { Station name } \\
\text { (owner) }\end{array}$ & $\begin{array}{l}\text { Station } \\
\text { location } \\
\left({ }^{\circ}\right)\end{array}$ & $\begin{array}{l}\text { Trigger } \\
\text { time }\end{array}$ & $\begin{array}{l}\text { S-minus } \\
\text { trigger } \\
\quad(s)\end{array}$ & $\begin{array}{l}\text { Direction } \\
(\mathrm{az})\end{array}$ & $\begin{array}{l}\text { Maximum } \\
\text { amplitude } \\
\text { (g) }\end{array}$ & $\begin{array}{l}\text { Duration } \\
(\mathrm{s})\end{array}$ \\
\hline \multirow{6}{*}{$\begin{array}{l}14 \text { December } 1988 \\
0016: 17.3 \text { G.m.t. } \\
\text { Eastern Calif. } \\
37.589 \mathrm{~N}, 118.847 \mathrm{~W} \\
\text { Magnitude } 2.9 \mathrm{MD}\end{array}$} & $\begin{array}{l}\text { McGee Creek } \\
\text { Crowley Lake } \\
\text { (USGS) (Triaxial) }\end{array}$ & $\begin{array}{r}37.550 \mathrm{~N} \\
118.811 \mathrm{~W}\end{array}$ & $16: 19.8^{4}$ & $4 \quad$ (2) & & (1) & \\
\hline & $\begin{array}{l}\text { McGee Creek } \\
\text { Crowley Lake } \\
\text { (USGS) (Multi-channel) }\end{array}$ & $\begin{array}{r}37.550 \mathrm{~N} \\
118.811 \mathrm{~W}\end{array}$ & $16: 19.8^{4}$ & $4 \quad$ (2) & & & \\
\hline & $166 \mathrm{~m}$ Downhole & & & - & & (1) & \\
\hline & $35 \mathrm{~m}$ Downhole & & & & & (1) & \\
\hline & $1 \mathrm{~m}$ Downhole & & & & & (1) & \\
\hline & Surface & & & & & (1) & \\
\hline \multirow{7}{*}{$\begin{array}{l}16 \text { December } 1988 \\
0553: 04.9 \text { G.m.t. } \\
\text { Southern Calif. } \\
33.980 \mathrm{~N}, 116.680 \mathrm{~W} \\
\text { Magnitude } 4.8 \mathrm{ML}\end{array}$} & $\begin{array}{l}\text { San Bernardino Array } \\
5931 \mathrm{~N} \text {. F Street } \\
\text { (USGS) }\end{array}$ & $\begin{array}{r}34.183 \mathrm{~N} \\
117.295 \mathrm{~W}\end{array}$ & $53: 23.7$ & (2) & & (1) & \\
\hline & $\begin{array}{l}\text { Forest Falls } \\
\text { Post office } \\
\text { (USGS) }\end{array}$ & $\begin{array}{r}34.088 \mathrm{~N} \\
116.919 \mathrm{~W}\end{array}$ & $53: 13.6$ & (2) & & (1) & \\
\hline & $\begin{array}{l}\text { Morongo Valley } \\
\text { Fire Station } \\
\text { (USGS) }\end{array}$ & $\begin{array}{r}34.048 \mathrm{~N} \\
116.577 \mathrm{~W}\end{array}$ & (3) & 2.1 & $\begin{array}{l}135 \\
\text { Up } \\
045\end{array}$ & $\begin{array}{l}.05 \\
.07 \\
.11\end{array}$ & $\begin{array}{l}0.0 \\
0.0 \\
1 \text { peak }\end{array}$ \\
\hline & $\begin{array}{l}\text { Cabazon } \\
\text { Post Office } \\
\text { (USGS) }\end{array}$ & $\begin{array}{r}33.918 \mathrm{~N} \\
116.782 \mathrm{~W}\end{array}$ & $53: 08.0$ & 1.2 & $\begin{array}{l}270 \\
\text { Up } \\
180\end{array}$ & $\begin{array}{l}.06 \\
.11 \\
.09\end{array}$ & $\begin{array}{l}0.0 \\
0.2 \\
0.0\end{array}$ \\
\hline & $\begin{array}{l}\text { Whitewater Canyon } \\
\text { Trout Farm } \\
\text { (USGS) }\end{array}$ & $\begin{array}{r}33.989 \mathrm{~N} \\
116.655 \mathrm{~W}\end{array}$ & (3) & (2) & $\begin{array}{l}270 \\
\text { Up } \\
180\end{array}$ & $\begin{array}{l}.15 \\
.14 \\
.12\end{array}$ & $\begin{array}{l}1.0 \\
1.7 \\
0.1\end{array}$ \\
\hline & $\begin{array}{l}\text { Anza Array, Garner } \\
\text { Valley Fire Station } \\
\text { (USGS) }\end{array}$ & $\begin{array}{r}33.616 \mathrm{~N} \\
116.627 \mathrm{~W}\end{array}$ & $53: 12.3$ & 5.1 & & (1) & \\
\hline & $\begin{array}{l}\text { Anza Array } \\
\text { Pine Meadow Ranch } \\
\text { (USGS) }\end{array}$ & $\begin{array}{r}33.578 \mathrm{~N} \\
116.589 \mathrm{~W}\end{array}$ & $53: 13.1$ & 5.5 & & (1) & \\
\hline $\begin{array}{l}4 \text { October 1987- } \\
21 \text { December } 1988 \\
\text { Southern Cal if. } \\
\text { Epicenter and } \\
\text { magnitude unknown }\end{array}$ & $\begin{array}{l}\text { Valyermo } \\
\text { Forest Station } \\
\text { (USGS) } \\
\text { Note: Probably }\end{array}$ & $\begin{array}{r}34.44 \mathrm{~N} \\
117.85 \mathrm{~W}\end{array}$ & rnia ever & ent of 3 & December & (1) & \\
\hline
\end{tabular}


Catalogue of Mational Cooperative Strong-Motion Network

accelerograph records recovered during 1988--Continued

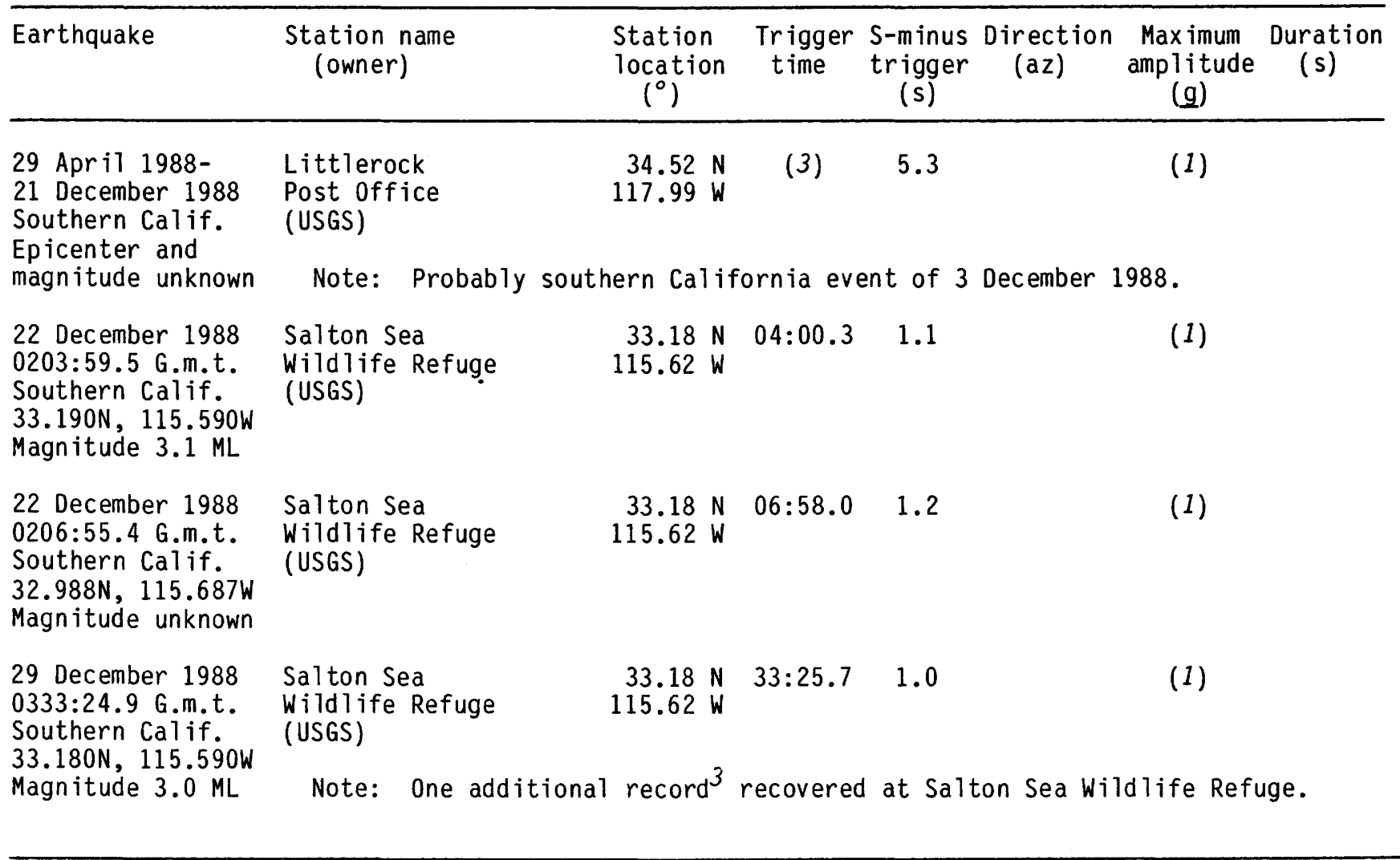

1 Less than $0.05 \mathrm{~g}$ at ground-level or less than $0.10 \mathrm{~g}$ at non-ground-level stations.

2 Questionable or indeterminable.

3 World Wide Voice Broadcast (WWVB) time code $i 1$ legible, or instrument not equipped with a radio receiver; correlation of accelerogram with event may be questionable or identity of event unknown.

4 Internal clock time; accuracy is variable. 



\section{SELECTED SERIES OF U.S. GEOLOGICAL SURVEY PUBLICATIONS}

\section{Perlodicals}

Earthquakes \& Volcanoes (issued bimonthly).

Preliminary Determination of Epicenters (issued monthly).

\section{Technical Books and Reports}

Professional Papers are mainly comprehensive scientific reports of wide and lasting interest and importance to professional scientists and engineers. Included are reports on the results of resource studies and of topographic, hydrologic, and geologic investigations. They also include collections of related papers addressing different aspects of a single scientific topic.

Bulletins contain significant data and interpretations that are of lasting scientific interest but are generally more limited in scope or geographic coverage than Professional Papers. They include the results of resource studies and of geologic and topographic investigations; as well as collections of short papers related to a specific topic.

Water-Supply Papers are comprehensive reports that present significant interpretive results of hydrologic investigations of wide interest to professional geologists, hydrologists, and engineers. The series covers investigations in all phases of hydrology, including hydrogeology, availability of water, quality of water, and use of water.

Circulars present administrative information or important scientific information of wide popular interest in a format designed for distribution at no cost to the public. Information is usually of short-term interest.

Water-Resources Investigations Reports are papers of an interpretive nature made available to the public outside the formal USGS publications series. Copies are reproduced on request unlike formal USGS publications, and they are also available for public inspection at depositories indicated in USGS catalogs.

Open-File Reports include unpublished manuscript reports, maps, and other material that are made available for public consultation at depositories. They are a nonpermanent form of publication that may be cited in other publications as sources of information.

\section{Maps}

Geologic Quadrangle Maps are multicolor geologic maps on topographic bases in 7 1/2-or 15-minute quadrangle formats (scales mainly $1: 24,000$ or $1: 62,500$ ) showing bedrock, surficial, or engineering geology. Maps generally include brief texts; some maps include structure and columnar sections only.

Geophysical Investigations Maps are on topographic or planimetric bases at various scales; they show results of surveys using geophysical techniques, such as gravity, magnetic, seismic, or radioactivity, which reflect subsurface structures that are of economic or geologic significance. Many maps include correlations with the geology.

Miscellaneous Investigations Series Maps are on planimetric or topographic bases of regular and irregular areas at various scales; they present a wide variety of format and subject matter. The series also includes 7 1/2-minute quadrangle photogeologic maps on planimetric bases which show geology as interpreted from aerial photographs. Series also includes maps of Mars and the Moon.
Coal Investigations Maps are geologic maps on topographic or planimetric bases at various scales showing bedrock or surficial geology, stratigraphy, and structural relations in certain coal-resource areas.

Oil and Gas Investigations Charts show stratigraphic information for certain oil and gas fields and other areas having petroleum potential.

Miscellaneous Field Studies Maps are multicolor or black-andwhite maps on topographic or planimetric bases on quadrangle or irregular areas at various scales. Pre-1971 maps show bedrock geology in relation to specific mining or mineral-deposit problems; post-1971 maps are primarily black-and-white maps on various subjects such as environmental studies or wildemess mineral investigations.

Hydrologic Investigations A tlases are multicolored or black-andwhite maps on topographic or planimetric bases presenting a wide range of geohydrologic data of both regular and irregular areas; principal scale is $1: 24,000$ and regional studies are at $1: 250,000$ scale or smaller.

\section{Catalogs}

Permanent catalogs, as well as some others, giving comprehensive listings of U.S. Geological Survey publications are available under the conditions indicated below from the U.S. Geological Survey, Books and Open-File Reports Section, Federal Center, Box 25425, Denver, CO 80225. (See latest Price and Availability List.)

"Publications of the Geological Survey, 1879-1961" may be purchased by mail and over the counter in paperback book form and as a set of microfiche.

"Publications of the Geological Survey, 1962- 1970" may be purchased by mail and over the counter in paperback book form and as a set of microfiche.

"Publications of the U.S. Geological Survey, 1971-1981" may be purchased by mail and over the counter in paperback book form (two volumes, publications listing and index) and as a set of microfiche.

Supplements for 1982, 1983,1984, 1985, 1986, and for subsequent years since the last permanent catalog may be purchased by mail and over the counter in paperback book form.

State catalogs, "List of U.S. Geological Survey Geologic and Water-Supply Reports and Maps For (State)," may be purchased by mail and over the counter in paperback booklet form only.

"Price and Availabllity List of U.S. Geological Survey Publications," issued annually, is available free of charge in paperback booklet form only.

Selected copies of a monthly catalog "New Publications of the U.S. Geological Survey" av ailable free of charge by mail or may be obtained over the counter in paperback booklet form only. Those wishing a free subscription to the monthly catalog "New Publications of the U.S. Geological Survey" should write to the U.S. Geological Survey, 582 National Center, Reston, VA 22092.

Note.--Prices of Government publications listed in older catalogs, announcements, and publications may be incorrect. Therefore, the prices charged may differ from the prices in catalogs, announcements, and publications. 
